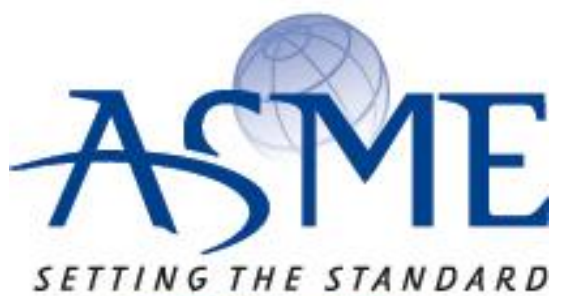

SETTING THE STANDARD

\section{American Society of Mechanical Engineers}

ASME Accepted Manuscript Repository

Institutional Repository Cover Sheet

ASME Paper Title: Implicit Discontinuous Galerkin Solution on Unstructured Mesh for Turbine Blade Secondary Flov

Authors: $\quad$ M. Yao and L. He

ASME Journal Title: Journal of Turbomachinery

Volume/Issue: $142 / 1$

Date of Publication (VOR* Online): December 14, 2019

https://asmedigitalcollection.asme.org/turbomachinery/article-

ASME Digital Collection URL: abstract/142/1/011004/1070437/Implicit-Discontinuous-Galerkin-Solution-on?redirect

DOI: $\quad$ https://doi.org/10.1115/1.4045551

Copyright (C) 2019 by ASME. CC-BY distribution license.

*VOR (version of record) 


\title{
Implicit Discontinuous Galerkin Solution on Unstructured Mesh for Turbine Blade Secondary Flow
}

\author{
M. Yao \\ Department of Engineering Science \\ University of Oxford \\ Oxford OX2 OES, UK \\ e-mail: min.yao@eng.ox.ac.uk \\ L. He \\ Department of Engineering Science \\ University of Oxford \\ Oxford OX2 OES, UK
}

\section{ABSTRACT}

To enhance solution accuracy with high order methods, an implicit solver using the Discontinuous Galerkin (DG) discretization on unstructured mesh has been developed. The DG scheme is favored chiefly due to its distinctive feature of achieving a higher order accuracy by simple internal sub-divisions of a given mesh cell. It thus can relieve the burden of mesh generation for local refinement. The developed method has been assessed in several test cases. Firstly an examination on the mesh-dependency with different DG orders for discretization has demonstrated the expected mesh convergence-order of accuracy correlation. The flow around a cylinder solved with up to the $5^{\text {th }}$ order accuracy demonstrates the need for a high order geometrical representation corresponding to the high-order flow field discretization. A calculated turbulent flat plate boundary layer demonstrates the capability of the high order DG in capturing the laminar sub-layer for a given coarse mesh $(y+>20)$ without a wall function, in a 
clear contrast to a conventional $2^{\text {nd }}$ order scheme. The focal test case of the present work is a high pressure (HP) turbine cascade with the flow losses being strongly influenced by both the laminar separation induced transition on the blade suction surface and the secondary flow development in the endwall region. Fully turbulent RANS solutions consistently over-predict the losses regardless, which can be significantly reduced by an empirically specified transition at a mid-chord point. Of particular interest is a contrasting behavior of pure laminar flow solutions. A steady laminar flow solution is difficult to converge, and even when converging, tends to give an unrealistically large separation. On the other hand when the laminar solution is run in a time accurate unsteady mode, a qualitatively different flow pattern emerges. Separated shear layer is then shown to lead to coherently shed unsteady vortices with a net entrainment from the main stream to near-wall region. The resultant time-averaged near-wall flow then effectively becomes a reattached boundary layer. Both overall and distributed losses computed from unsteady laminar solutions are shown to be consistently much better than the fully turbulent RANS solutions. It is remarkable and quite unexpected that for such a seemingly complex 3D flow problem, the straightforward unsteady laminar solutions with no empirical input seem to be comparable to (or slightly better than) the tripped RANS with empirical input.

\section{INTRODUCTION}

Computational fluid dynamics (CFD) has played a significant role in turbomachinery design and analysis in past few decades. However, the conventional CFD methods as widely applied are mostly based on $2^{\text {nd }}$ order discretization schemes, which tend to be dissipative when high resolution of practical turbomachinery problems is pursued. Recent development in applications of high fidelity flow models has 
indicated the requirement for developing and applying new high order methods. Highorder schemes based on finite difference upwind-biased weighted essentially nonoscillatory (WENO), e.g. [1] tend to be limited to structured grids and require rather large neighboring stencils for higher order discretization. Another high-order finite volume method (FVM) using an upwind flux scheme with Monotonic UpstreamCentered Scheme for Conservative Law (MUSCL) reconstruction has been applied for predicting the transitional flow over turbine cascade [2] while the extension to highorders is also restrictive.

On the other hand, there exists a family of high-order discretization schemes following a distinctively different strategy of achieving the high-order accuracy totally relying on local internal elements without extensive stencils on extra mesh points. The feature of internal sub-division is particularly appealing given the difficulties in generating quality fine meshes for complex geometries. Among these high-order schemes, the DG discretization has gained huge interest in recent years due to its very nature of easy extension to high-orders by increasing the order of piece-wise polynomials and maintaining the conservation laws without restrictions of inter-element continuity. DG is firstly introduced to solve the neuron transport problems [3] and further extended to solve linear hyperbolic problems. The breakthrough of solving the non-linear hyperbolic equations, particularly the compressible Euler equations, in a general framework is developed [4], which employs a Total Variation Diminishing (TVD) to obtain the high-order temporal discretization. The extension to the Navier-Stokes equations using a DG method is first made by Bassi and Rebay [5] introducing the BR1 
scheme to treat the discretization of viscous terms and the BR2 scheme is further put forward to eliminate the extended stencils. A stable flux reconstruction (FR) method, recovering the nodal DG scheme, is more recently proposed [6].

The DG approach has been extensively applied to external aerodynamics, and also started to be developed and applied to internal turbomachinery flows, though to a much less extent. The DG method solving the Reynolds-Averaged Navier-Stokes (RANS) with the k- $\omega$ model has been applied to simulate the main flow features of turbomachinery flow [7-9].

High-fidelity turbulence eddy resolved (instead of modelled) methods, like large eddy simulation (LES), have been applied to study the endwall flows in the context of low pressure turbine (LPT) cascade [10-12]. Turbulence eddy resolved simulations have also been conducted more recently using the DG based methods, typically at relatively low Reynolds numbers. The direct numerical simulation (DNS) using a DG discretization has been lately applied to a LPT cascade to capture the flow separation and transition at low Reynolds numbers [13, 14], on a two dimensional (2D) geometry with a small spanwise length without considering the endwall effects. Another preliminary study of LES of a full-span LPT cascade with the DG by introducing an extra wall-resolved model has been carried out [15], but only has the benchmark test case of a turbulent channel flow been validated. An entropy-stable DG spectral-element method has also been employed to simulate a LPT cascade $[16,17]$ with only blade profile loss being taken into account, again due to lack of the endwall effects. 
It is worth pointing out that most previous high order DG studies have been confined to 2D (quasi 3D) configurations. It is well known that a major source of flow losses in a blade passage, particularly in high pressure turbines (HPT), is in the endwall region dominated by secondary flows [18]. To the authors' knowledge, the endwall secondary flow development in a 3D HPT blade passage is yet to be studied by a highorder scheme.

Furthermore, it is of the present interest to examine a 3D turbine configuration where flow losses are influenced by both the secondary flow in the endwall region and the blade surface laminar flow separation with a subsequent transition. The instabilities of the separated laminar shear-layer subsequently lead to a transition to a turbulent state followed by a reattachment. For a RANS based flow solver, the boundary layer transition can be treated by 2D boundary layer based transition models from very simple separation bubble length correlated models e.g. [19], and those more sophisticated transport-equation based transitional models [20]. It is noted that strong $3 \mathrm{D}$ secondary flow effects in the endwall region should challenge those 2D boundary layer based transition models. The lack of high-order DG solutions for the flows with largely 2D blade surface boundary layer separation interacting with 3D endwall secondary flows also provides a motivation to examine the applicability of a DG solver to the complex 3D flow interactions.

Given the background introduced above, the present study has been initiated to develop an implicit high-order DG solver on unstructured meshes for turbomachinery applications. An implicit formulation seems to be suited for an unstructured mesh 
solver, for which some well-established solution techniques for explicit methods (e.g. multigrid methods) are less effective.

In the following sections, the DG methodology will be presented first. Some basic validation and sensitivity studies are followed to illustrate the distinctive features and characteristics of the DG method. Flow losses in a HP turbine cascade subject to the influences of the secondary flow and the laminar separation will be examined and compared to the corresponding experimental data. Finally, some further considerations on the modelling of the related flow physics are presented and the corresponding observations are made.

\section{NUMERICAL METHOD}

\subsection{Discontinuous Galerkin Discretization}

The computational domain $\Omega$ is partitioned into a set of non-overlapping elements $k$ with boundary $\partial \Omega$. The approximate solutions $\mathbf{U}_{h}(\mathbf{x}, t)$ to the flow equations in space $\mathbf{x} \in \Omega$ and time space $t \in[0, T]$ belong to finite $3 \mathrm{D}$ space $\mathbf{V}_{h}$ are sought.

$$
\begin{aligned}
\frac{\partial \mathbf{U}}{\partial t}+\nabla \cdot\left(\mathbf{F}^{c}(\mathbf{U})-\mathbf{F}^{v}(\mathbf{U}, \nabla \mathbf{U})\right) & =\mathbf{S}(\mathbf{U}, \nabla \mathbf{U}) \\
\mathbf{F}^{v}-\mathbf{A} \nabla \mathbf{U} & =0
\end{aligned}
$$

where an auxiliary equation Eqn. (1b) is included to obtain the first order system equations. Here, the viscous flux $\mathbf{F}^{v}$ in an form of the state variables and gradients of 
state variables is defined, $\mathbf{F}^{v}=\mathbf{A} \nabla \mathbf{U}$, where $\mathbf{A}=\frac{\partial \mathbf{F}^{v}(\mathbf{U}, \nabla \mathbf{U})}{\partial \nabla \mathbf{U}}$ is the Jacobian matrix of viscous flux.

To obtain the approximate solution $\mathbf{U}_{h}$, the governing equation Eqn. (1a) is multiplied by a test function $\mathbf{v}_{h} \in \mathbf{V}_{h}$ and integrated over the domain. Then, the DG weak formulation is achieved by finding $\mathbf{U}_{h} \in \mathbf{V}_{h}$, such that all $\mathbf{v}_{h} \in \mathbf{V}_{h}$,

$$
\int_{k} \mathbf{v}_{h}^{T} \frac{\partial \mathbf{U}_{h}}{\partial t} d \mathbf{x}+\mathbf{R}_{h, C}\left(\mathbf{U}_{h}, \mathbf{v}_{h}\right)+\mathbf{R}_{h, V}\left(\mathbf{U}_{h}, \mathbf{v}_{h}\right)+\mathbf{R}_{h, S}\left(\mathbf{U}_{h}, \mathbf{v}_{h}\right)=0
$$

where $\mathbf{R}_{h, C}, \mathbf{R}_{h, V}$ and $\mathbf{R}_{h, S}$ represent the discretized inviscid flux, viscous flux and source terms. Since the turbomachinery flows are always mix of incompressible and compressible flows, the inviscid advection upstream splitting method (AUSM+UP) flux designed for all-speed flows is adopted [21], which combines the efficiency from the flux-vector splitting and the accuracy from the flux-difference splitting methods with enhanced robustness. In order to maintain the compactness of a high-order scheme, the viscous flux is discretized using the BR2 scheme. For turbulence closure, the one equation Spalart-Allmaras (SA) turbulence model is fully coupled with the flow equations, and further modified to avoid the negative working variables and prevent the oscillations when applying a high-order discretization across the discontinuity [22].

\subsection{Basis Functions}

The approximate solution and test functions are represented as the expansions of $\mathrm{p}$-th order polynomials,

$$
\mathbf{U}_{h}(\mathbf{x}, t)=\sum_{i=1}^{N} \hat{\mathbf{U}}_{i}(t) b_{i}(\mathbf{x})
$$




$$
\mathbf{v}_{h}(\mathbf{x})=\sum_{j=1}^{N} \hat{\mathbf{v}}_{j} b_{j}(\mathbf{x})
$$

where the $\hat{\mathbf{U}}_{i}(t)$ and $\hat{\mathbf{v}}_{j}$ stand for the expansion coefficients and $b_{i}(\mathbf{x})$ and $b_{j}(\mathbf{x})$ denote the basis shape functions with $i, j=\{1, \ldots, N\}$, where $N$ is the number of degrees of freedom. For example, the number of mesh nodes to cover a 2D triangular mesh cell is $N=(p+1)(p+2) / 2$, as shown in Fig. 1 . The order of accuracy in the spatial discretization corresponds the order of the polynomial, which is also denoted as such in the DG notation. Thus DG1 $\left(p=1, N=3\right.$, Fig.1a) is of $2^{\text {nd }}$ order accuracy, and DG3 $(p=3, N=10, F i g .1 c)$ is of $4^{\text {th }}$ order accuracy. Noted that the expansions are performed within each elements without any continuity constraints on the boundaries with adjacent elements, which distinguishes the DG method from a standard Galerkin method. In addition, the basis function $b_{j}(\mathbf{x})$ in the physical elements $k$ is transformed from the basis function in the reference elements $\hat{k}$ through the mapping functions.

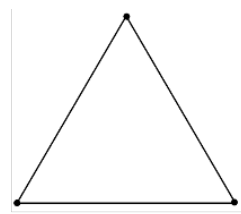

a) DG1 ( $2^{\text {nd }}$ order)
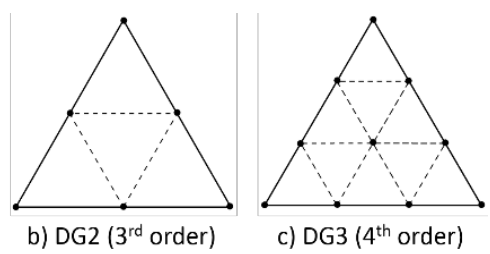

Fig. 1 A 2D mesh cell with different orders of internally divided sub-cells (indicated by dash lines)

\subsection{Implicit Time Integration}

The spatially discretized equations can be integrated easily in time using an explicit scheme such as the multi-stage Runge-Kutta scheme [4]. The time step size of the explicit scheme is restricted by the corresponding small CFL, normally less than 1 , due to the numerical stability requirement. 
An implicit temporal integration scheme is developed as a primary solution method in the present work. The implicit scheme has an advantage of using very large time steps (CFL $O\left(10^{3}\right)$ in the present work). Given the challenges of developing effective multi-grid methods for unstructured meshes, the implicit formulation proves to be an important way of accelerating solutions for both steady and unsteady flows. It is worth noting that for very complex geometry configurations, highly non-uniform meshes might be generated. The time step limit set by the minimum mesh size of a highly non-uniform and also highly skewed unstructured mesh can be too restrictive, particularly for unsteady flow solutions where a uniform time step needs to be taken for the temporal consistency.

The spatial discretization in Eqn. (2) leads to a system of ordinary differential equations (ODE) in time,

$$
\mathbf{M} \frac{d \hat{\mathbf{U}}}{d t}+\mathbf{R}=0
$$

where $\mathbf{M}$ is the global block-diagonal mass matrix including all the mass matrices of elements. $\hat{\mathbf{U}}=\left(U_{1}, U_{2}, \ldots, U_{k}, \ldots\right)^{T}$ is the global vector of expansion coefficients, where $U_{k}$ represents the expansion coefficients vector of $k$-th element. And $\mathbf{R}=\left(R_{1}, R_{2}, \ldots, R_{k}, \ldots\right)^{T}$ denotes the global vector of residuals, where $R_{k}$ is the residual vector of $k$-th element. Next, the Euler backward scheme is employed to solve the system ODE in time,

$$
\mathbf{M} \frac{\mathbf{U}^{n+1}-\mathbf{U}^{n}}{\Delta t}+\mathbf{R}\left(\mathbf{U}^{n+1}\right)=0
$$


where the superscript $n$ represents the time step level. Since residuals are non-linear terms in the governing equations, Eqn. (5) is a non-linear system of algebraic equations. For the sake of resolving this kind of non-linear system equations, the linearization of residuals is performed,

$$
\mathbf{R}\left(\mathbf{U}^{n+1}\right)=\mathbf{R}\left(\mathbf{U}^{n}\right)+\frac{\partial \mathbf{R}\left(\mathbf{U}^{n}\right)}{\partial \mathbf{U}^{n}}\left(\mathbf{U}^{n+1}-\mathbf{U}^{n}\right)
$$

Note that the global Jacobian matrix $\partial \mathbf{R}(\mathbf{U}) / \partial \mathbf{U}$ is a $N_{e} \times N_{e}$ block matrix, where $N_{e}$ is the number of total elements in the whole computational domain. For the local element $k$, the local block matrix is a $m N \times m N$ Jacobian matrix, where $m$ is the number of conservative flow variables.

The non-linear system Eqn. (5) can be solved using a Newton method as in the following iterative steps,

1. Newton time step $k=0, \mathbf{U}^{(0)}=\mathbf{U}^{n}$

2. For $k=1, \ldots, \infty$

$$
\underbrace{\left(\frac{\mathbf{M}}{\Delta t}+\frac{\partial \mathbf{R}\left(\mathbf{U}^{(k)}\right)}{\partial \mathbf{U}^{(k)}}\right)}_{\mathbf{A}} \underbrace{\left(\mathbf{U}^{(k+1)}-\mathbf{U}^{(k)}\right)}_{\mathbf{x}}=\underbrace{-\left(\frac{\mathbf{M}}{\Delta t}\left(\mathbf{U}^{(k)}-\mathbf{U}^{n}\right)+\mathbf{R}\left(\mathbf{U}^{(k)}\right)\right)}_{\mathbf{b}}
$$

3. $\mathbf{U}^{n+1}=\left.\mathbf{U}^{(k)}\right|_{k \rightarrow \infty}$

At time level $n+1$, the Newton iteration is initialized with a previous time level solution $\mathbf{U}^{n}$ and iterations are performed to reach the sufficient small convergence tolerance as $\mathbf{U}^{n+1}=\left.\mathbf{U}^{(k)}\right|_{k \rightarrow \infty}$. In each Newton iteration, $\mathbf{A x}=\mathbf{b}$ is solved using a preconditioned GMRES iterative algorithm with a block incomplete LU factorization with zero fill-in (ILU0) [23]. Compared to other preconditioning schemes like block Jacobian or block 
Gauss-Seidel, ILUO is the most robust one with a smaller number of iterations especially when applied to the low Mach flows on highly stretched meshes.

The overall DG discretization and implicit temporal integration process is illustrated in Fig. 2, where three nested loops are included. The outermost loop is the time step loop, following by the inner Newton iterations, while the GMRES iterative scheme sits in the innermost loop.

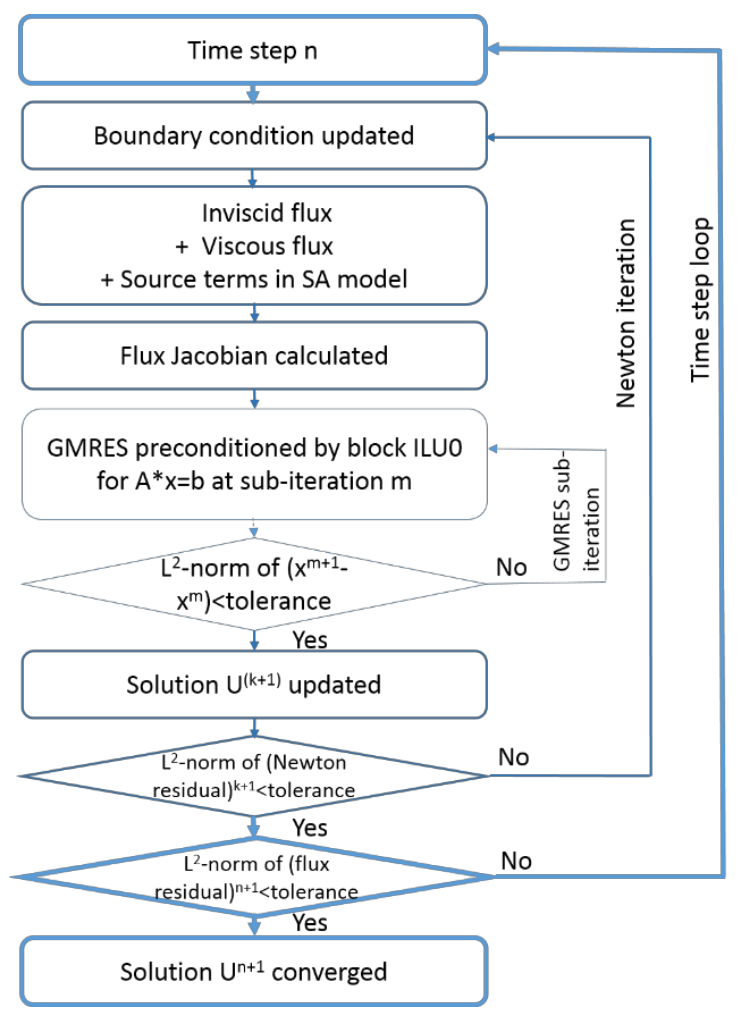

Fig. 2 DG discretization and implicit time integration process

\subsection{Boundary Condition}

The boundary conditions are applied through the inviscid and viscous flux across the physical boundary in a weak manner. These fluxes are computed based on the interior state and gradient of state and a constructed exterior boundary state. A list of typical physical boundary conditions are imposed as follows. A subsonic inflow boundary 
condition can be specified with four incoming Riemann invariants, e.g. via total pressure $p_{0}$, total temperature $T_{0}$ and inflow angle $\alpha$, with one extrapolated outgoing Riemann invariant. The S-A turbulence working variable $\tilde{v}$ also needs to be given here if the S-A turbulence model is used in RANS simulations. A subsonic outflow boundary condition is specified using one incoming Riemann invariant with four outgoing Riemann invariants. Thus, the static pressure $p$ is imposed whilst other state variables are extrapolated from the interior domain.

For the no-slip wall, the velocity and eddy viscosity are all prescribed to zero and an adiabatic condition is also imposed here requiring that the heat flux is set to zero in the energy equation. For a slip wall boundary condition, the flow is tangential to the wall. A far-field boundary condition requires that the boundary is far away enough from the solid body and the boundary state is specified by given freestream variables.

\section{COMPUTATIONAL RESULTS}

This section covers three validation cases, $2 \mathrm{D}$ inviscid and viscous flows around a cylinder, a 2D turbulent flat plate boundary layer and a 3D high pressure turbine cascade.

\subsection{Flow around a cylinder}

The first validation study is an inviscid flow around a cylinder with an inflow velocity at $35 \mathrm{~m} / \mathrm{s}$. The geometry of cylinder is $2 \mathrm{D}$ but a 3D mesh is generated by extruding the 2D geometry one layer in the third direction to accommodate the current 3D solver. The mesh generator adopted is Gmsh [24], an open-source software for 
generating a 3D finite element mesh. The physical mesh near the cylinder surface is shown in Fig. 3(a) and there are only 12 node points to represent the geometry of the cylinder. The effect of using a high-order boundary representation is examined by comparing high-order DG simulations with such a coarse wall geometry mesh. Figs. 3(b), 3(c) and 3(d) exhibit high-order curved boundary with iso-parametric elements represented by corresponding $p$-th order polynomials as adopted in the spatial discretization [25]. The $\mathrm{p}$-th order of the boundary polynomials is denoted by "BPp". The DG flow field simulations by $p$-th order polynomials are denoted as "DGp", where the corresponding order of the discretization accuracy is $p+1$.

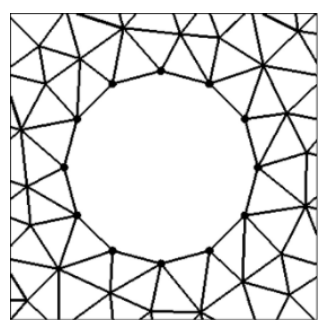

a) BP1

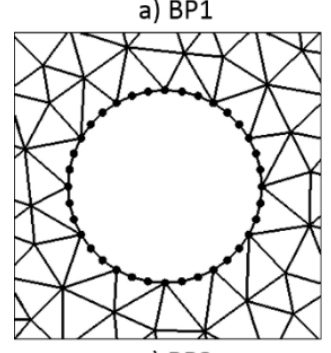

c) BP3

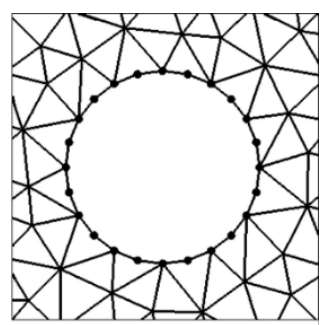

b) $\mathrm{BP} 2$

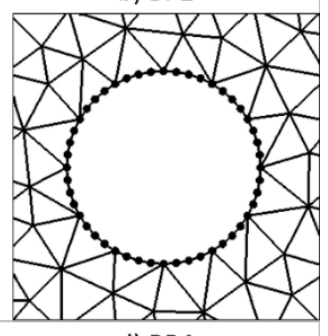

d) BP4

Fig. 3 High-order geometry representations of cylinder with 12 base-mesh points

The static pressure coefficient $\mathrm{Cp}$ contours from the high-order curved boundary representations consistent with the DG discretization orders are given in Fig. 4. It is clearly shown that flow region is better resolved as the order of accuracy increases with the corresponding order of polynomials representing the boundary. The computed surface $\mathrm{Cp}$ distribution is also compared with the theoretical exact solution, in Fig. 5. 
The theoretical surface $\mathrm{Cp}$ distribution is given by $C p=1-4 \sin ^{2} \theta$ where the stagnation points locate at $\theta=0, \pi$. For this coarse mesh, DG0 and DG1 solutions are unable to match the theoretical one whilst all other results of higher order (DG2, DG3, DG4) agree very well with the exact solution.

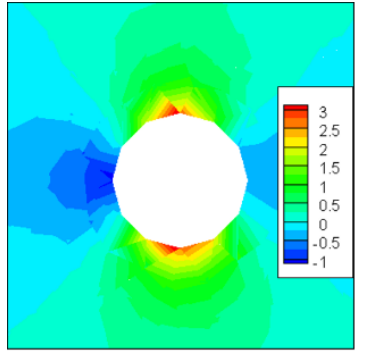

a) DG1 + BP1

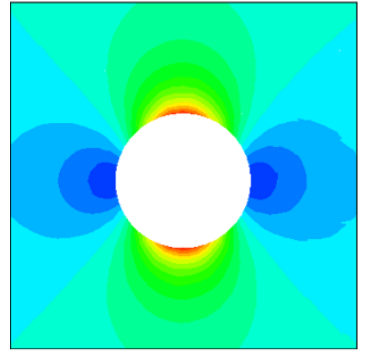

c) $\mathrm{DG} 3+\mathrm{BP} 3$

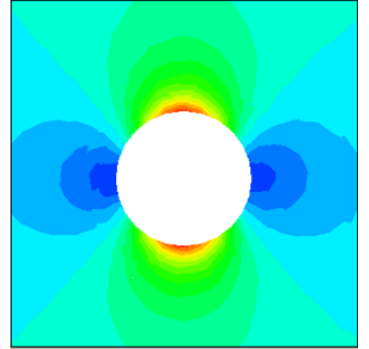

b) $D G 2+B P 2$

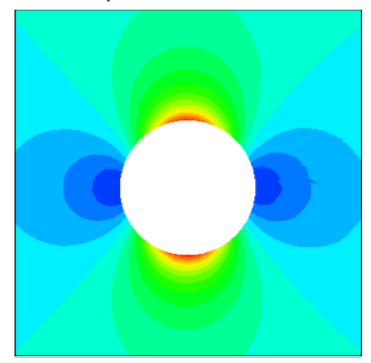

d) DG4 + BP4

Fig. 4 Static pressure coefficient contours for an inviscid flow around cylinder of DG representations with corresponding order of boundary geometrical representation

In contrast, a test with a linear boundary representation (BP1) combined with different DG orders of the discretization is also conducted. The corresponding pressure contours are shown in Fig. 6. The lower order (DG1 DG2) solutions tend to converge to a steady state, though not fully, and the results are very poor. The higher order (DG3 DG4) solutions on the other hand, are unable to converge to a steady state at all. Thus, we can see that a high-order field discretization may not be very meaningful without a corresponding high-order boundary geometrical representation. 


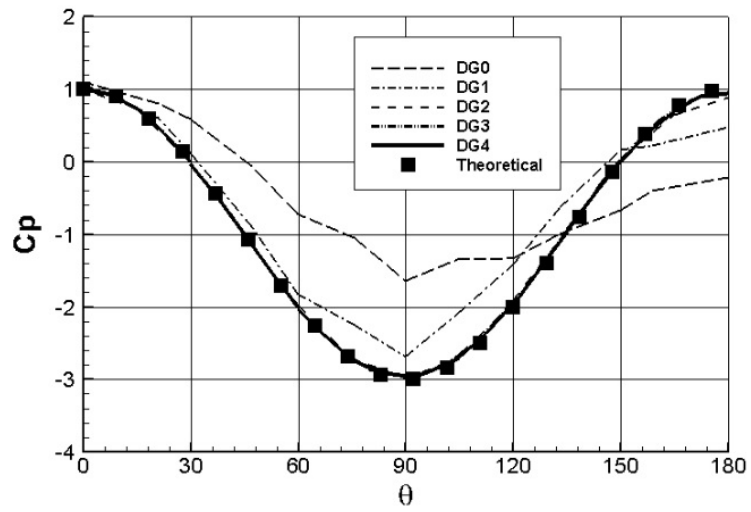

Fig. 5 Static pressure coefficient distributions around cylinder ( $\theta=0$ : leading edge;

$\theta=180^{\circ}:$ trailing edge)

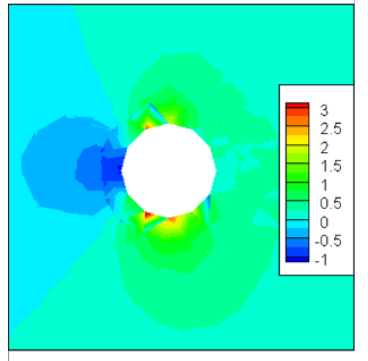

a) $D G 1+B P 1$

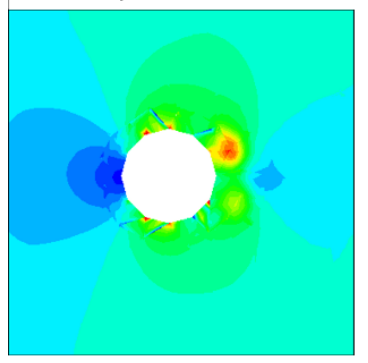

c) $D G 3+B P 1$

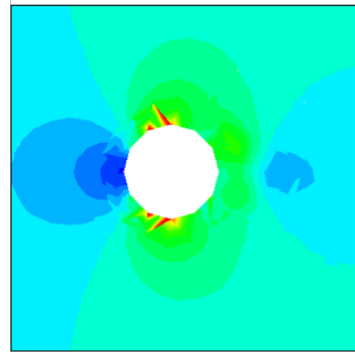

b) $D G 2+B P 1$

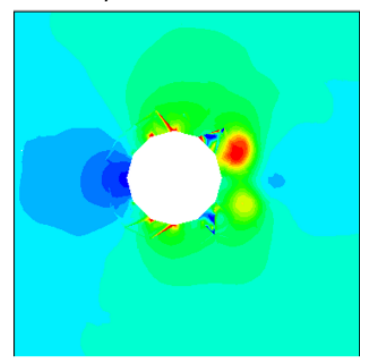

d) $D G 4+B P 1$

Fig. 6 Static pressure coefficient contours for an inviscid flow around cylinder with linear boundary representation (BP1) 


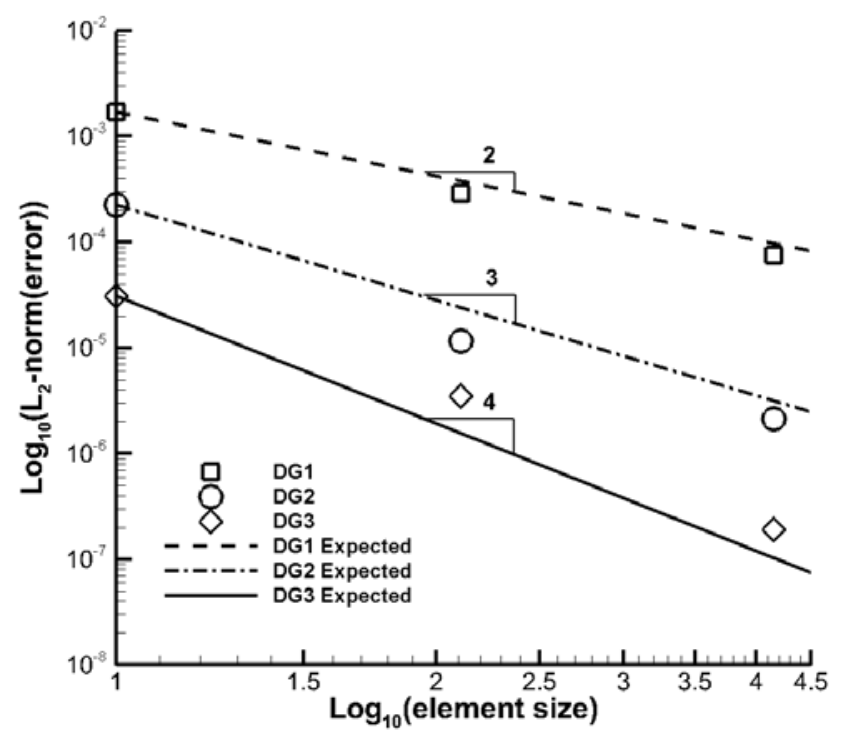

Fig. 7 Mesh-DG order convergence for DG1, DG2 and DG3 inviscid cylinder flow

To further quantitatively examine and demonstrate the accuracy of different orders of the DG solutions, the mesh is refined in both $x$ and $y$ coordinate directions twice by a factor of 1.5 for this $2 \mathrm{D}$ test case. The convergence rates of the inviscid flow around cylinder from DG1 to DG3 are shown in Fig. 7. It can be seen that the results of the different DG orders follow closely the expected ideal benchmarks.

The computational cost from DG1 to DG3 on the coarse mesh is given in Table 1. Here, the degrees of freedom for a 3D tetrahedral element are defined as $N=(p+1)(p+2)(p+3) / 6$. The $5 N \times 5 N$ Jacobian matrix needs to be calculated and inverted in each implicit time step, where the CPU time is increased significantly as the DG orders increase. It should be noted that at the current stage, the developed code is far from being optimized, indicated by the slower than expected scaling of the computational speed when the DG order is increased. 
Table 1. CPU time for DG1, DG2 and DG3 inviscid cylinder flow on coarse mesh

\begin{tabular}{|c|c|c|c|}
\hline & DG1 & DG2 & DG3 \\
\hline CPU Time (s) & 230.81 & 940.41 & 5552.75 \\
\hline CPU Time/CPU time from DG1 & 1.0 & 4.1 & 24.1 \\
\hline
\end{tabular}

We then examine a laminar flow past a cylinder to validate unsteady viscous flow modelling capability. An inflow velocity is taken as $55 \mathrm{~m} / \mathrm{s}$, and the Reynolds number is 174 based on the free stream velocity and the diameter of cylinder. For this case the experimental data are available from the early work of Thom [26], which is also recently used for validation purposes [27]. The mesh refined in the near wall region for capturing the laminar boundary layer is shown in Fig. 8 . There are still only 12 node points to represent the cylinder geometry and so the high-order boundary representation is also adopted for high-order DG simulations. In Fig. 9, the instantaneous entropy contours are presented for the solutions from a $2^{\text {nd }}$ order DG1 to a $5^{\text {th }}$ order DG4. As clearly shown in Fig. 9, the shed vortices tend to be better resolved and less dissipated as the order of accuracy is increased. Except for the DG1, the calculated Strouhal (St.) numbers from the DG2 to the DG4 are consistently close to 0.186 , in good agreement with the empirical value around 0.19 for this Reynolds number. 


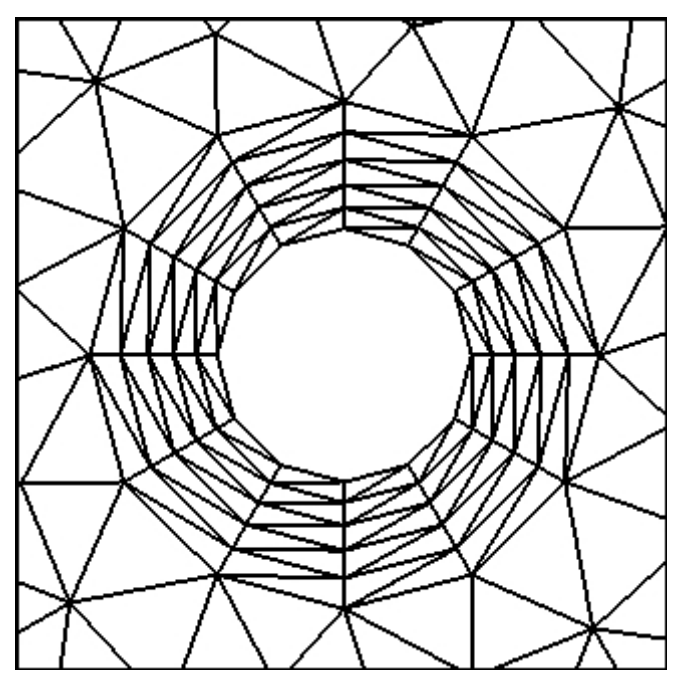

Fig. 8 Mesh around cylinder for unsteady laminar solution

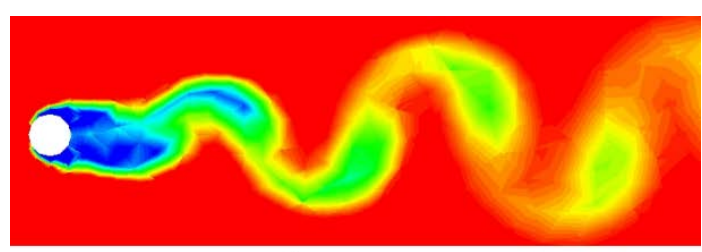

a) DG1 + BP1

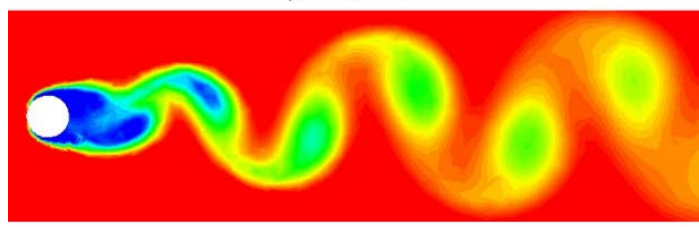

b) $D G 2+B P 2$

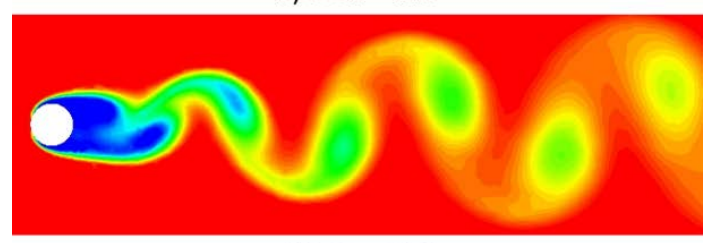

c) $D G 3+B P 3$

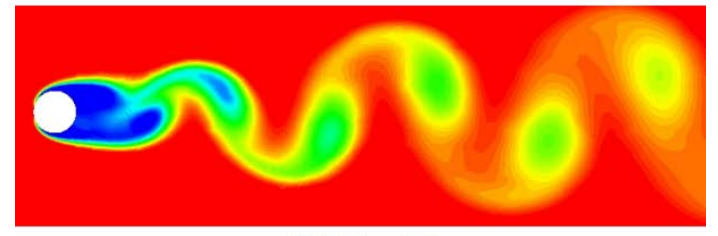

d) DG4 + BP4

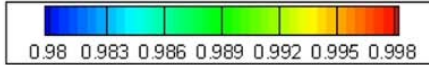

Fig. 9 Instantaneous entropy [exp(-S/R)] contours for cylinder flow 
The time-averaged surface pressure coefficients from the DG1 to the DG4 solutions are plotted in Fig. 10. For this case, sufficiently higher order DG solutions (DG3 and DG4) are in good agreement with each other and with the experimental data.

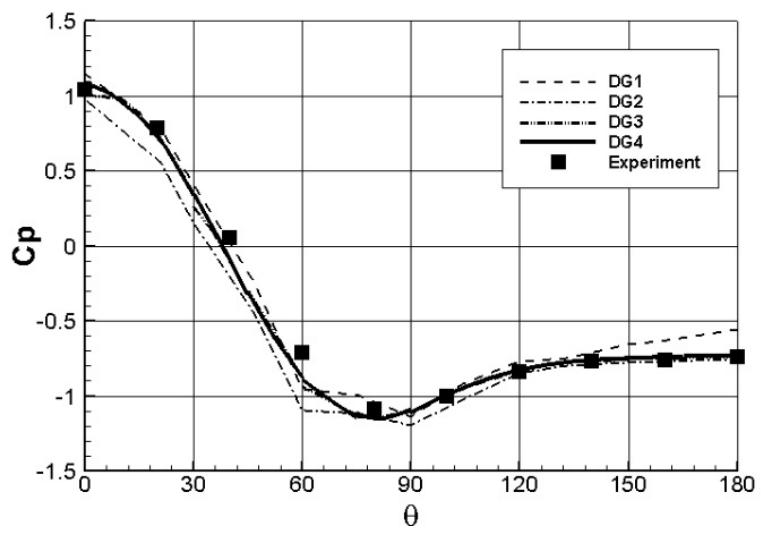

Fig. 10 Time-averaged pressure coefficients on cylinder surface ( $\theta=0$ : leading edge; $\theta=180^{\circ}$ : trailing edge), compared to experiment data of Thom [26]

\subsection{Turbulent Boundary Layer over Flat Plate}

The second test case is for a turbulent boundary layer over a flat plate with an inflow velocity $34.6 \mathrm{~m} / \mathrm{s}$ and a Reynolds number of $6 \times 10^{6}$. Fig. 11 displays the 3D computational mesh ( $26 \times 15 \times 3$, the numbers of nodes in $x, y, z$ directions) extruding from a $2 \mathrm{D}$ one with a periodic boundary condition in the third direction. The first element space normal to the wall is $\Delta y / l=1.2 \times 10^{-4}$ with $l$ denoting as the length of flat plate and a mesh spacing stretching ratio is 1.78 . The first element from the leading edge along the wall is $\Delta x / l=5.2 \times 10^{-4}$ and a spacing ratio is chosen as 1.6 . This nearwall mesh would be extraordinarily coarse for a typical $2^{\text {nd }}$ order spatial discretization but it can be employed for high-order DG computations. 


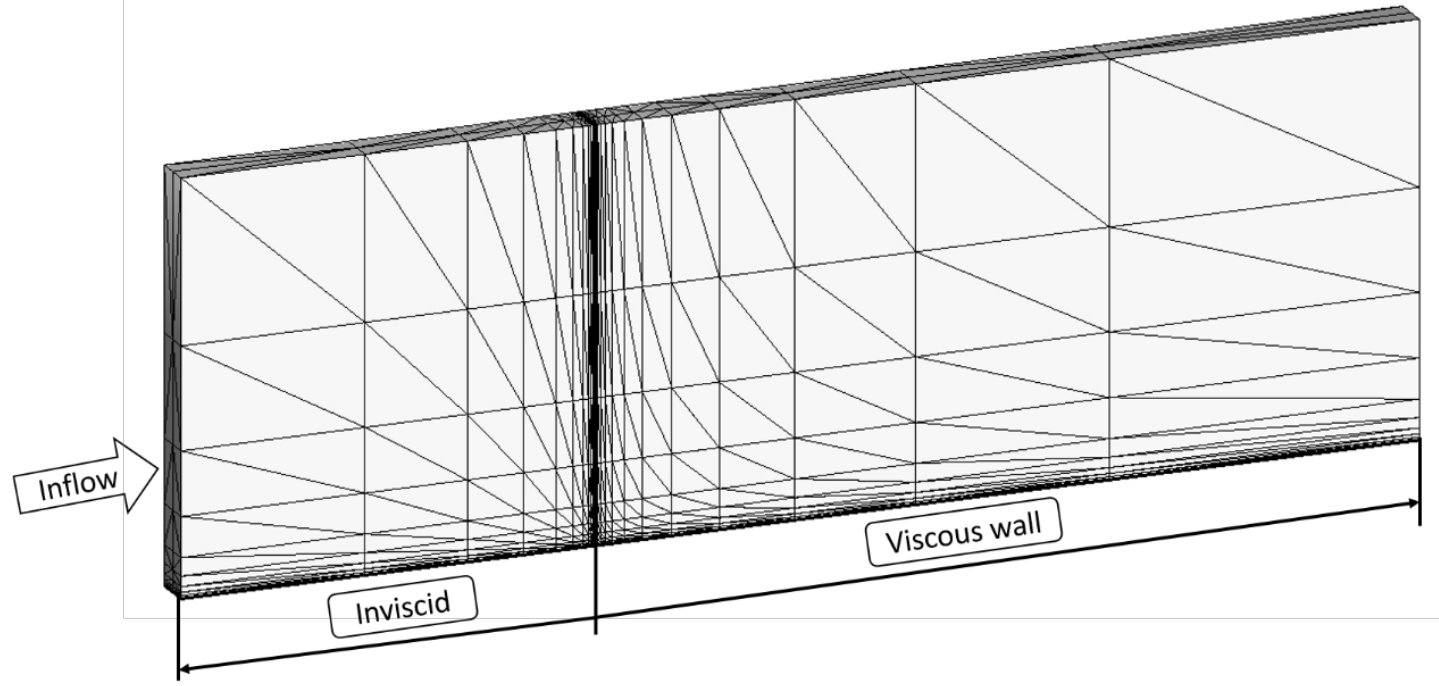

Fig. 11 Computed mesh and boundary condition for turbulent flat plate

To compare the near wall behavior computed with different orders of accuracy, the non-dimensional velocity $u^{+}$profiles against $y^{+}$are plotted in Fig. 12 for the DG solutions from the $2^{\text {nd }}$ order to the $5^{\text {th }}$ order, all with the direct nonslip wall condition. The first mesh element $y^{+}$for the $2^{\text {nd }}$ order solution is markedly higher than that typically require for a direct nonslip wall condition. It is nearly 20 and thus $u^{+}$is substantially over-predicted by the $2^{\text {nd }}$ order scheme for this mesh. The computed results get closer to the theoretical law of wall as the order of accuracy increases. Overall, the $5^{\text {th }}$ order (DG4) results match very well with the theoretical velocity profile in both the viscous sub layer and the log law layer. Hence, we can see that good results can be achieved by high-order DG solutions on extremely coarse meshes where a conventional $2^{\text {nd }}$ order scheme would struggle. It must be reminded that all these solutions of different DG orders are obtained on the same physical mesh. The results thus also serve as a demonstration that the burden of generating high quality near wall 
fine meshes for complex geometries may be considerably alleviated by the high order methods for turbulent flow simulations without invoking the wall function.

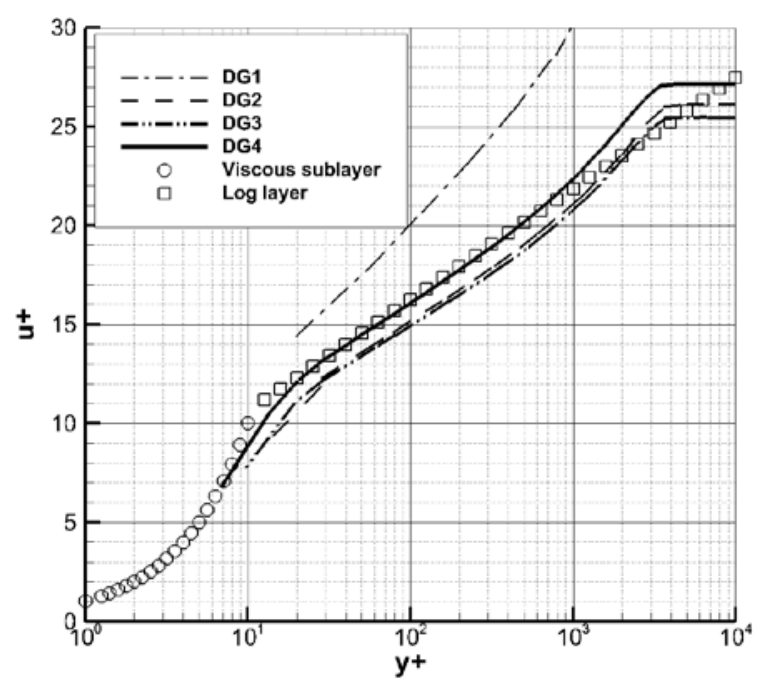

Fig. 12 Computed velocity profiles of turbulent boundary layer at $\operatorname{Re}_{x}=6 \times 10^{6}$

\subsection{High Pressure Turbine Cascade}

As stated in the introduction, one primary motivation of the present work is to examine the predictability of 3D blade aerodynamic performance influenced by both endwall secondary flow and blade surface bubble type separation. To this end, the high pressure turbine cascade experimentally studied at Durham University (commonly known as 'Durham Cascade', [28]) is selected.

\subsubsection{Durham Turbine Cascade and RANS Turbulent Flow Solutions}

Durham cascade has been used extensively in the past for studying secondary flow loss generation and optimization of enwall geometry for loss reduction, and also as a standard test case for validating turbomachinery CFD methods [29-32]. At the 
experimental conditions with a Reynolds number of $4 \times 10^{5}$, the flow in the blade passage is shown to be transitional subject to a bubble type separation on suction surface [30].

The main parameters are given in Table 2. Durham HPT cascade has a high flow turning angle, approximately $110^{\circ}$, typical for HPT rotor blades. The large secondary flow developed through the cascade has been comprehensively investigated experimentally, with various measurements for velocities, flow angles and total pressure losses, acquired with a five-hole probe at different traverse slots [28]. The computed results at axial chord position of $C_{a x}=128 \%$ (i.e. $28 \%$ ) are compared in the present study. The boundary layer at the end wall is largely laminar and transition to turbulent boundary layer occurs on the suction surface near the trailing edge, while a laminar separation bubble in the midspan region has been clearly observed at approximately $80 \%$ axial chord on the suction surface [31]. To predict the secondary flow in the Durham cascade, several turbulence models have been attempted but the transitional flow effects have been shown to be challenging [33].

Table 2. Geometrical and Flow parameters of Durham turbine cascade

\begin{tabular}{cc}
\hline \hline Inlet angle & $42.75^{0}$ \\
Outlet angle & $68.7^{0}$ \\
Reynolds Number & $4 \times 10^{5}$ \\
Exit Mach number & 0.1 \\
\hline
\end{tabular}

The present DG solver in the RANS mode has been applied to predict the main flow and secondary loss. This case has been firstly attempted with a fully turbulent 
solution with the Sparlart-Allamas turbulence model, as presented in [34]. Some of the preliminary fully turbulent RANS results are presented here as a reference point.

A 2D blade mesh is firstly made and then extruded in the third direction, approximately 50,000 node points, 300,000 mesh cells are generated using Gmsh for the RANS simulations of this half-span blade passage with one endwall, where $\Delta y^{+}<0.7$ on the blade surface and $\Delta z^{+}<0.5$ on the end wall.

The pitchwise mass-averaged total pressure loss coefficient $\mathrm{Cp} 0$ at $128 \% C_{a x}$ is presented in Fig. 13. Clearly the agreement between the DG1 and the DG2 solutions shows a reasonable order of accuracy convergence, and both show a clear overprediction of flow losses, which is consistent with previous RANS predictions [33, 35].

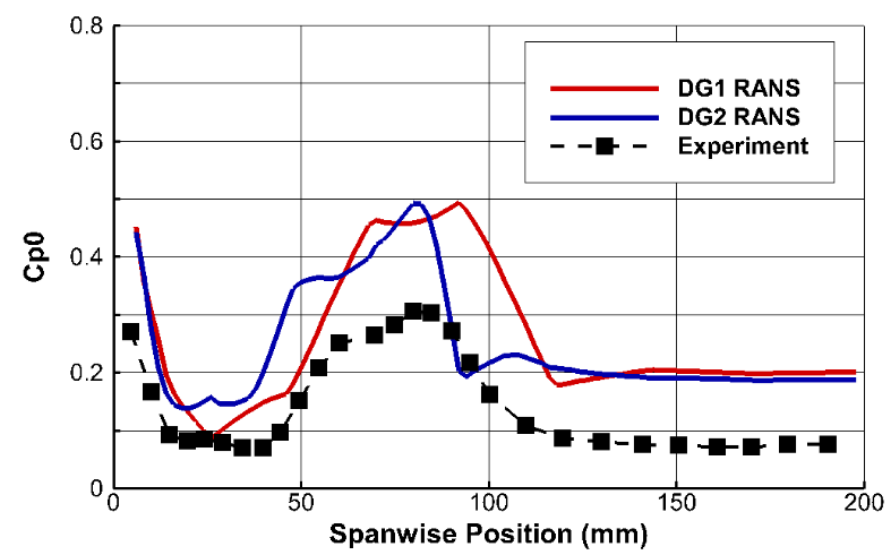

Fig. 13 Pitchwise mass-averaged Cp0 (Exp vs RANS) at $128 \% C_{a x}$

Given the knowledge that the boundary layer on the blade suction surface is transitional subject to a laminar separation at about $70 \%-80 \%$ chord, further RANS simulations with artificially tripped transition set at $70 \% C_{a x}$ are carried out. The specified tripping is implemented by simply forcing a laminar flow solution with zero 
eddy viscosity until the specified axial position. The pitchwise mass-averaged $\mathrm{CpO}$ losses at $128 \% C_{a x}$ of the solution with the tripped transition are compared to the fully turbulent results as shown in Fig. 14. Now lower losses are produced by the tripped transition solutions, clearly indicating that the transitional flow nature seems to be the main reason for the significant loss over-prediction of the fully turbulent solutions. The detailed loss profiles of the tripped solutions still are quite different from the experimental results, which is hardly surprising given the very empirical nature of the tripping. Also noted is that for both the tripped and the fully turbulent solutions, the predicted losses in the mid span region are less sensitive to the DG orders than the secondary flow losses in the endwall region.

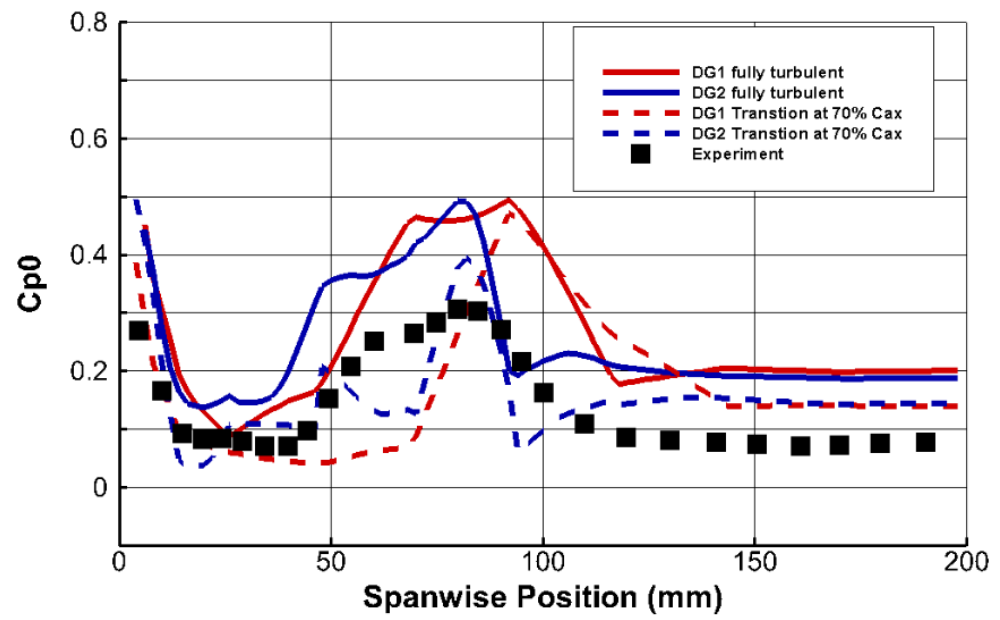

Fig. 14 Pitchwise mass-averaged Cp0 (RANS vs RANS with transition) at $128 \% C_{a x}$

\subsubsection{Some Further Modeling Considerations}

The flow losses predicted by the steady turbulent RANS solutions lead to further modelling considerations, even with a recognition that adoption of an established transition RANS model (not attempted in the present work) may reduce the empiricism 
of the tripping. A basic issue of interest is whether or not a steady flow model is adequate. A steady laminar flow solution is firstly attempted and the DG1 solution is shown in Fig. 15. The solution struggles to converge to a steady state, but the results reveal two distinctive but also physical sources of the problem, firstly a laminar bubble type separation starts at about $80 \%$ suction surface, and secondly vortices shed from the blunt trailing edge. Therefore, there are two parts of the flow where the role of unsteadiness needs to be considered:

a) Oscillatory shear layer of the laminar separation;

b) Unsteady vortex shedding from the blade trailing edge.
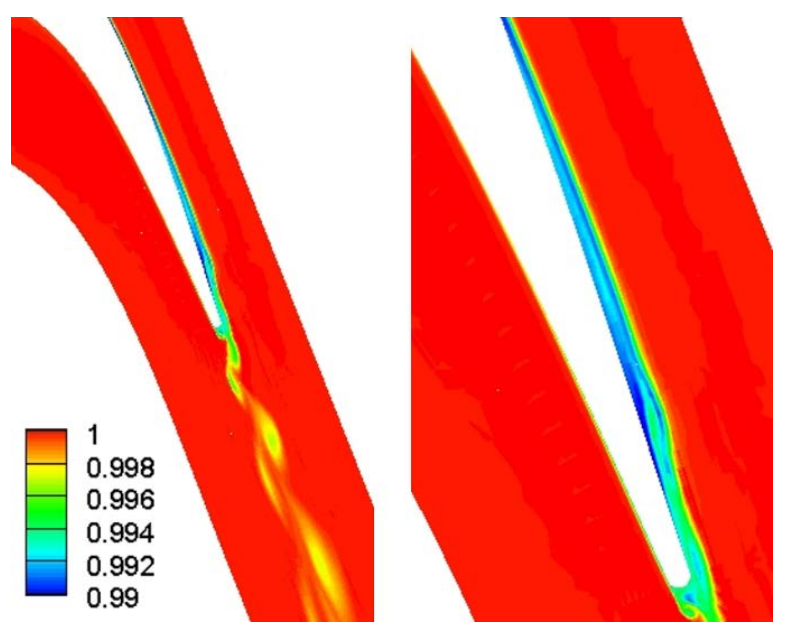

Fig. 15 Entropy contours of steady laminar solution from DG1: (a) overall domain and (b) close-up of suction surface near trailing edge

Both phenomena can be adequately captured by a full LES, but a much finer mesh typically by a factor of $O\left(10^{2}\right)$ larger than that for a RANS would then be needed. It is of interest to explore what solution behavior one might get when using a coarse mesh typical of RANS solutions. It is recognized that the bubble type separation can be captured by certain transition models [20], however the behavior of a boundary layer 
based transition model in a 3D secondary flow strongly influenced by the endwall is rather unknown. Furthermore, a RANS model, even with a properly captured transition on the blade surface, will be ultimately limited by the unknown interplay between the modeled turbulence unsteadiness (via an eddy viscosity) and the resolved unsteady shed vortices. The local self-excited unsteady vortices become both modelled and resolved at the same time, thus 'double-counted', with modelling uncertainty. The potential 'double counting' turbulence can be reduced if there is clearly spectral gap in the unsteadiness. For a blade trailing edge, it is unclear if there is such an easily definable spectral gap between relatively short-scale vortex shedding and large scale turbulence eddies, particularly those originated from the upstream shear layer instabilities of the laminar separation.

For a transitional RANS, once turbulent flow is tripped (by a transitional model or a simple tripping), the turbulence eddy viscosity convected to the trialing-edge will impact on the onset and evolution of the trailing edge unsteady vortex shedding, e.g. as observed by Ning and He [36]. Typical RANS based transition models are based on boundary layer flows, and it is unknown how these models would cope with unsteady flow around a blunt trailing edge. More specifically for the present case, if the transition is triggered by the suction surface laminar separation, a steady turbulent boundary layer should be predicted by the transition model. The trailing edge flow will then still be subject to turbulence eddy-viscosity convected from the upstream turbulent boundary layer, which might potentially suppress the onset and evolution of vortex shedding. It is 
known that the trailing edge vortex shedding strongly affects the trailing edge basepressure and thus the losses e.g. [18], [36], [37].

A further modeling consideration in the present work stems from a recent work by He and Yi [37], which indicates that an essentially unsteady laminar solution, even with seemingly much coarser temporal and spatial resolutions than a typical LES, may predict an effectively reattached separation bubble on a compressor blade, in agreement with the experiment. The key requirement seems to be an unsteady laminar solution with sufficient temporal resolution to capture the evolution of the large scale unsteady vortices in the separated shear layer. For the present case, we thus attempt a direct fully laminar flow model. Accordingly, a consistent numerical constraint in this present case is that the temporal and spatial resolution should be sufficiently fine to resolve both the evolution of the unstable laminar separated shear layer on the blade suction surface as well as that of the trailing edge vortex shedding.

\subsubsection{Sensitivity Study on Temporal Resolution of Unsteady Laminar Solution}

Given the above modelling consideration and intent, we need to first obtain some guidance on the corresponding temporal resolution required for an adequate unsteady laminar solution. A finer mesh has been generated to ensure that the spatial scales of the shear layer instability and the trailing edge vortex shedding are adequately captured, as shown in Fig. 16. For the shear layer instability, a typical RANS boundary layer mesh can only provide a reasonable resolution in the wall normal direction, so further refinement in the streamwise is added as indicated in Fig. 16 with a close up around the suction surface separation region. Similarly around the trailing edge and in 
the wake region, a sufficiently fine mesh is required to support the local resolution to capture the initiation and development of the vortex shedding as illustrated in Fig. 16 for the close up around the T.E. and wake. The total mesh cells for the half-span turbine blade passage is 1.2 million with $2 \times 10^{5}$ node points and this mesh resolution is still much coarser than one for a LES for the half-span passage domain. It is also pointed out that high-order DG simulations are equivalent to subdividing mesh points and increasing the DG order for a given mesh should have a similar effect to that of a mesh refinement. The periodic boundary conditions are applied at the pitch-wise boundaries of the computational domain. The endwall and mid-span are subject to the no-slip condition and the symmetric condition, respectively.

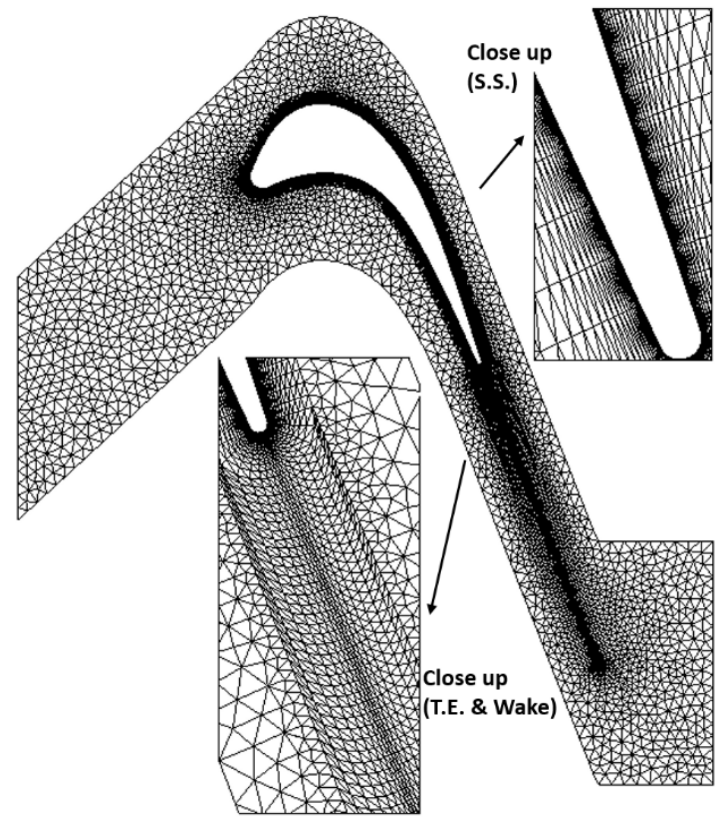

Fig. 16 Mesh for direct laminar simulations

Temporal resolution requirement can then be examined by the comparative study in terms of number of time steps in one period of the unsteady shed vortices to be 
resolved. Here, the calculations are conducted for the 2D turbine cascade based on the midspan flow conditions. Three time steps corresponding to three maximum CFL numbers (denoted $\mathrm{CFL}_{\mathrm{m}}$ ) of 1000, 500 and 250 are examined. For this Durham cascade, only are the DG1 ( $2^{\text {nd }}$ order) and DG2 ( $3^{\text {rd }}$ order) computations carried out. Fig. 17 shows the instantaneous entropy contours in the near trailing edge region from the DG1 and DG2 on the three different maximum CFL numbers ( $C F L_{m}=1000,500$ and 250).

Apart from the large time step case $\left(C F L_{m}=1000\right)$, both the DG1 and DG2 solutions for $C F L_{m}=500$ and 250 have captured shear layer instabilities and convective transport of unsteady disturbances to downstream. More specifically, the DG2 solution shows better resolved vortical structures than the DG1, as a higher-order discretization results in less numerical dissipation. The number of time steps per period and Strouhal number for the trailing edge shedding vortices are compared in Table 3. The computed time steps per one period and Strouhal numbers for the DG1 and DG2 at $\mathrm{CFL}_{\mathrm{m}}=500$ and 250 are very consistent. Note that the present predicted HP turbine cascade vortex shedding Strouhal number is close to the value of 0.235 in the VKI turbine blade as computed by Ning and He [36].

It is observed that the computed unsteady characteristics will be largely consistent once that the number of time steps for the one period of vortex shedding at the trailing edge is about 80 . The shed vortices from the shear layer of the suction surface separation has a nearly twice longer period, the number of time steps per period of the suction surface unsteady separation is approximately $160-180$, corresponding to a DG1 result of $\mathrm{CFL}_{m}=500$. For comparison purposes, the number of 
time steps for a blade passage domain through-flow time is about 4800 . It must be recognized that both the mesh counts and the time step sizes taken here are by a factor of $O\left(10 \sim 10^{2}\right)$ coarser than those typical for LES.

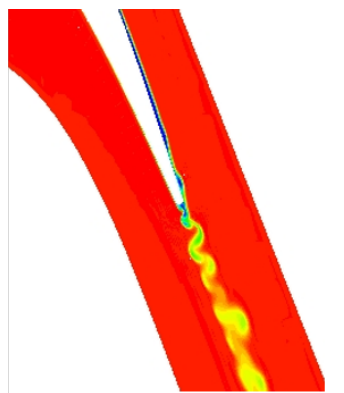

a) $\mathrm{DG} 1 \mathrm{CFL}_{\mathrm{m}}=1000$

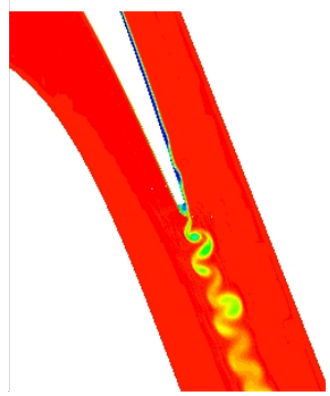

c) $\mathrm{DG} 1 \mathrm{CFL}_{\mathrm{m}}=500$

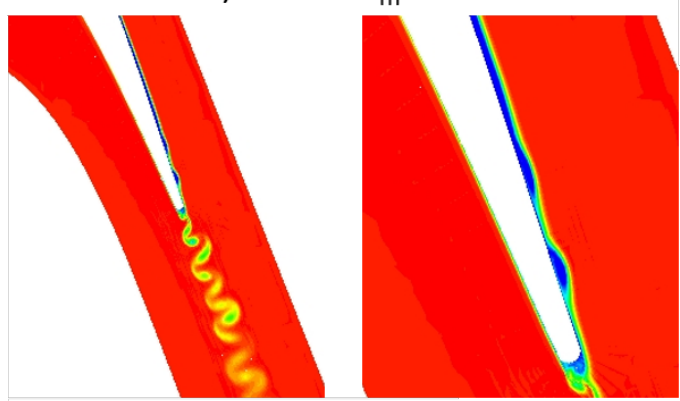

e) DG1 $\mathrm{CFL}_{\mathrm{m}}=250$
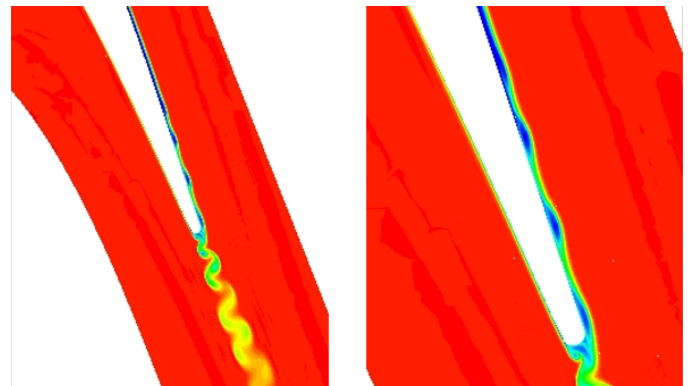

b) $\mathrm{DG} 2 \mathrm{CFL}_{\mathrm{m}}=1000$
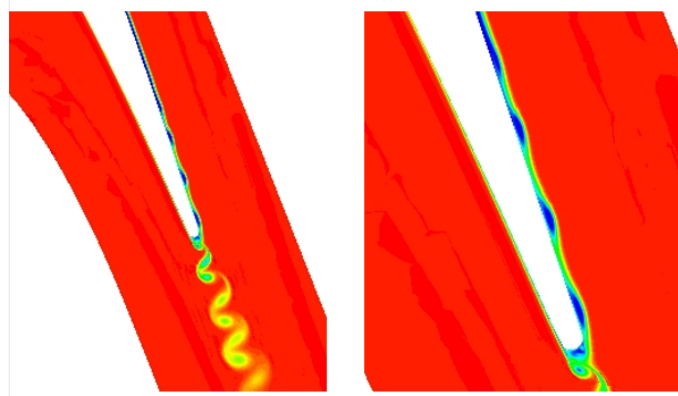

d) $\mathrm{DG} 2 \mathrm{CFL}_{\mathrm{m}}=500$

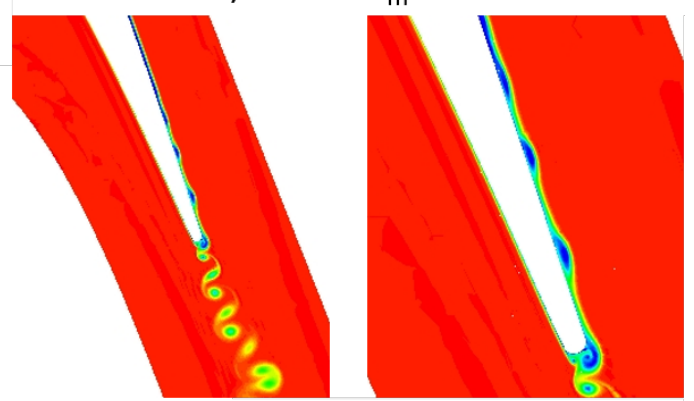

f) $\mathrm{DG} 2 \mathrm{CFL}_{\mathrm{m}}=250$

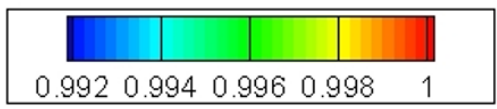

Fig. 17. Instantaneous entropy contours near suction surface and trailing edge for $C F L_{m}=$ 1000, 500 and 250. 
Table 3. Numbers of time steps per period and calculated St. numbers for trailing edge vortex shedding

\begin{tabular}{|c|c|c|c|c|c|c|}
\hline \multirow{2}{*}{} & \multicolumn{2}{|c|}{ CFLm $=1000$} & \multicolumn{2}{c|}{ CFLm $=500$} & \multicolumn{2}{c|}{ CFLm $=250$} \\
\cline { 2 - 7 } & DG1 & DG2 & DG1 & DG2 & DG1 & DG2 \\
\hline Time steps & 39 & 37 & 78 & 78 & 151 & 151 \\
\hline St. number & 0.220 & 0.233 & 0.219 & 0.221 & 0.226 & 0.228 \\
\hline
\end{tabular}

The unsteady solutions are time averaged over a few passage through-flow time to obtain the time-averaged parameters. Given the importance of base pressure in loss generation around a trailing edge, we may look at the local time-averaged static pressure contours from the DG1 and DG2 solutions at $C F L_{m}=1000,500$ and 250 , as shown in Fig. 18. A steady laminar solution is also presented in Figs. 18(a) and 18(e) for comparison. The steady solutions miss the vortex shedding, as clearly observed. They thus only predict a high pressure region, resulting from the flow reattachment from both suction and pressure surfaces. The unsteady time-averaged solutions even at a large time step (CFLm $=1000$, Figs. 18(b) and 18(f)), still qualitatively capture the vortex shedding, leading to apparently different results from the steady one. Moreover, the unsteady solutions with increased temporal resolution at $C F L_{m}=500$ and $C F L_{m}=250$ (Figs. 18(c), 18(d), 18(g) and 18(h)) show that the pressure fields around the trailing edge are considerably more consistent. The results confirm that the time-step independent time mean solution can be reached when the maximum CFL number is less than 500 . 
Correspondingly there are 80 time steps per a trailing edge vortex shedding period for this case with $C F L_{m}=500$.

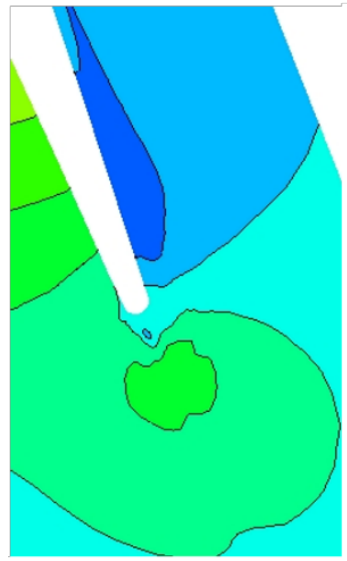

a) DG1 Steady

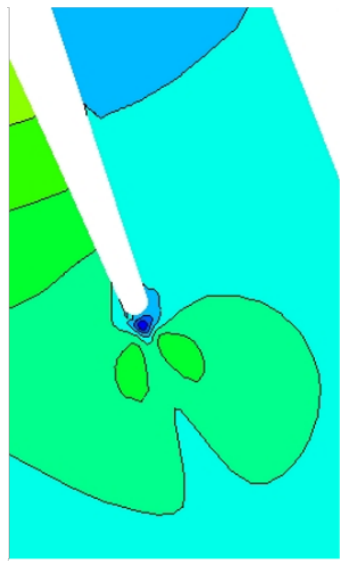

c) DG1 $\mathrm{CFL}_{m}=500$

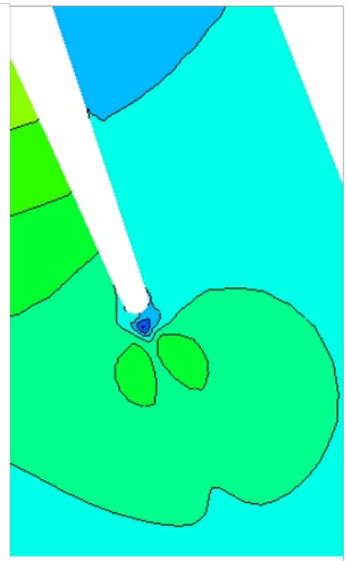

b) DG1 $\mathrm{CFL}_{\mathrm{m}}=1000$

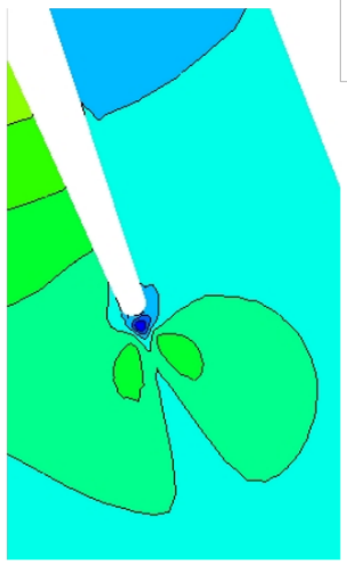

d) DG1 $\mathrm{CFL}_{m}=250$

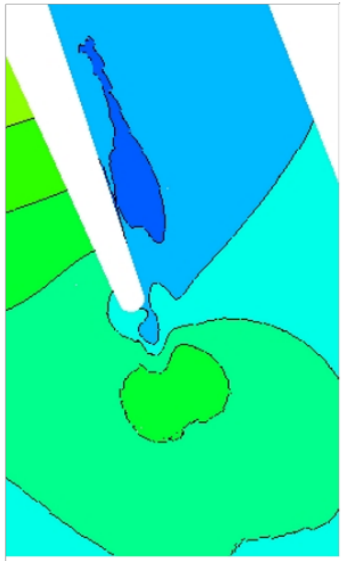

e) DG2 Steady

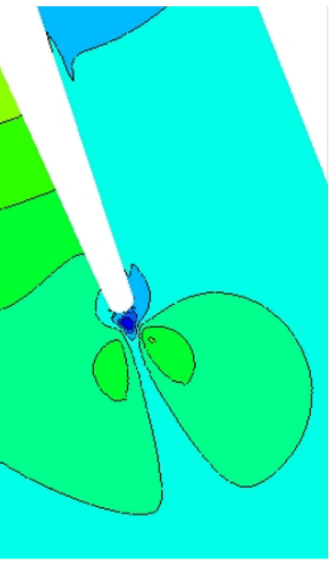

g) $\mathrm{DG} 2 \mathrm{CFL}_{\mathrm{m}}=500$

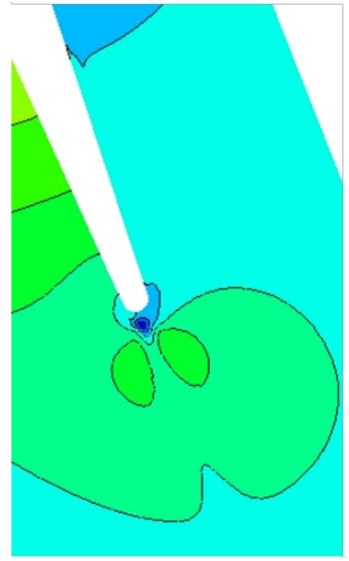

f) $\mathrm{DG} 2 \mathrm{CFL}_{\mathrm{m}}=1000$

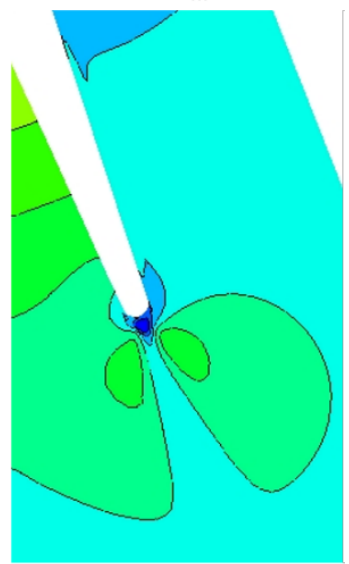

h) DG2 $\mathrm{CFL}_{\mathrm{m}}=250$

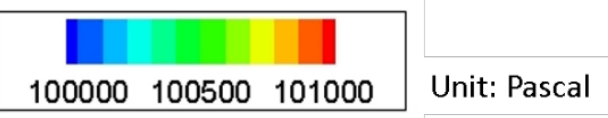

Fig. 18 Comparison of steady and unsteady time-averaged static pressure contours around trailing edge

As stated earlier, it is of interest to see if the large scale resolved unsteady shed vortices from the suction surface separation (e.g. as shown in Fig.17) would effectively 'reattach' the separated shear layer, to lead a reattached boundary layer like a turbulent boundary layer, as suggested in the previously results of $\mathrm{He}$ and $\mathrm{Yi}$ [37]. Fig. 19 displays 
the time-averaged Mach number contours from the steady and unsteady solutions at $\mathrm{CFL}_{\mathrm{m}}=500$.

Firstly, note the steady laminar solution clearly produces an unrealistic large laminar separation, as shown in Figs. 19(a) and 19(b). The consistent steady results by both the DG1 ( $2^{\text {nd }}$ order) and DG2 ( $3^{\text {rd }}$ order) also suggest that the separation flow behavior would be erroneously predicted by a steady flow model regardless of the spatial resolution. On the other hand, the time-averaged unsteady laminar solutions with the well resolved large scale unsteadiness (Figs. 19(c) and 19(d)), clearly show an effectively reattached time-mean boundary layer.

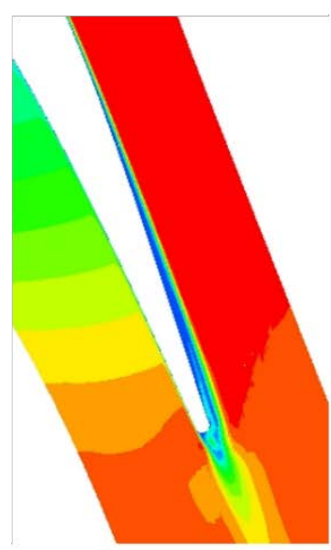

a) DG1 Steady

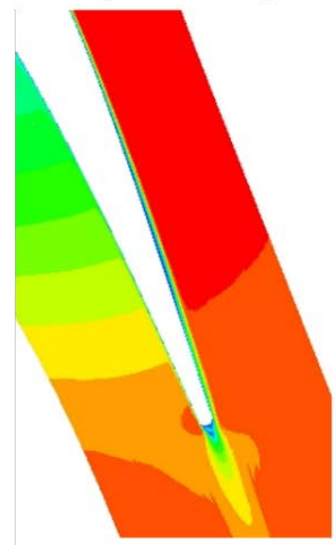

c) DG1 $\mathrm{CFL}_{\mathrm{m}}=500$

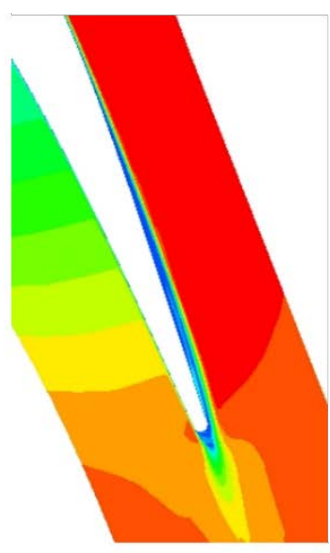

b) DG2 Steady

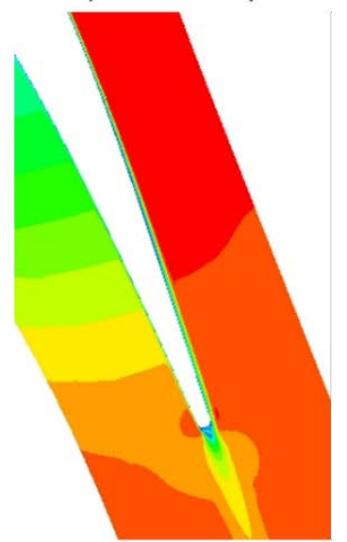

d) DG2 $\mathrm{CFL}_{\mathrm{m}}=500$

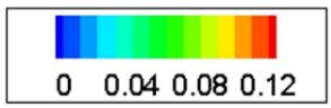


Fig. 19 Time-averaged Mach contours

The qualitatively contrasting characteristics between the steady and unsteady laminar flow solutions may be interpreted simply as shown in Fig. 20. For the steady laminar solution, the instabilities of separated shear-layers are suppressed by the lack of temporal resolution. A steady (or largely steady due to numerical convergence difficulties) flow field is then produced where the separated shear layers may eventually reattach, leading to a large separation bubble (Fig. 20a). On the other hand, for an unsteady solution, as long as the resolution is sufficient to capture the laminar shear layer instabilities, separated shear layers are unable to grow into a large vortex before being shed. Once a vortex is shed, the local flow becomes reattached before the separation starts another vortex. We thus have coherent unsteadily shed vortices (Fig. 20b). The small unsteady vortices also seem to generate a net entrainment of flow from the main stream to near-wall region. The time-averaged net flow effectively behave similarly to a reattached turbulent boundary layer.

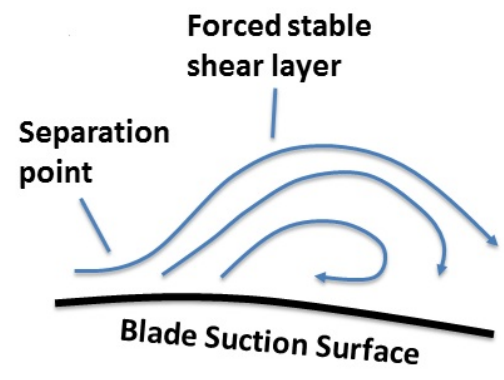

a) Large Separation Bubble (Steady Laminar Solution)

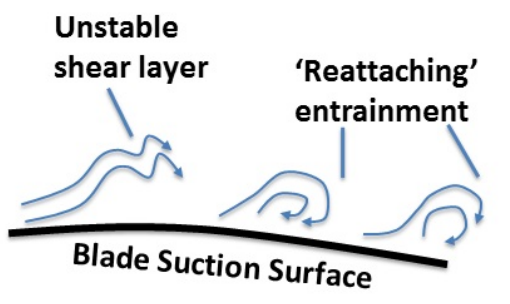

b) Small Unsteadily Shed Vortices (Unsteady Laminar Solution)

Fig. 20 Laminar Flow Patterns (Steady vs. Unsteady Solutions) 
The striking differences between the steady and unsteady laminar solution underline the marked importance of unsteadiness in the present case and other similar cases. Based on the findings of the present sensitivity study on the temporal resolution, all the subsequent 3D unsteady laminar calculations of the Durham HPT cascade are carried out with the time-step size corresponding to that of $\mathrm{CFL}_{m}=500$ for this $2 \mathrm{D}$ case.

\subsubsection{D Unsteady Laminar Solution of Durham Cascade}

We now look at the 3D unsteady laminar calculations of the Durham HP turbine cascade. The time averaged solutions are obtained over time steps to cover at least for one passage through-flow time. The time-averaged total pressure losses have been measured at downstream at a location of $128 \%$ axial chord $\left(\mathrm{x}=128 \% C_{a x}\right)$. We first look at the sensitivity of the DG solution with respect to the orders of accuracy.

The results of time-averaged total pressure loss coefficients $\mathrm{Cp} 0$ from the DG1 and DG2 solutions compared to the measured experiment data [38] are presented in Fig. 21. We can see that for the mesh adopted, the solutions become largely independent of the order of accuracy of the DG discretization. The measured data of the experiment illustrated in Fig. 21(a) show a passage vortex, a main counter vortex sits farther from the end wall while a small counter vortex is located in a crossflow region near the end wall. Now we compare the results between the time-averaged unsteady laminar results shown in Figs. 21(b) and 21(c) and the measure data of the experiment. The time-averaged unsteady laminar DG1 solution does not capture the passage vortex core well whereas the DG2 solution distinctively predicts this loss peak as well as the counter vortex located farther above the passage vortex. Both the DG1 and DG2 
solutions capture the small counter vortex close to the end wall. Overall, the total pressure loss of the DG2 solution is slightly better than that of the DG1.

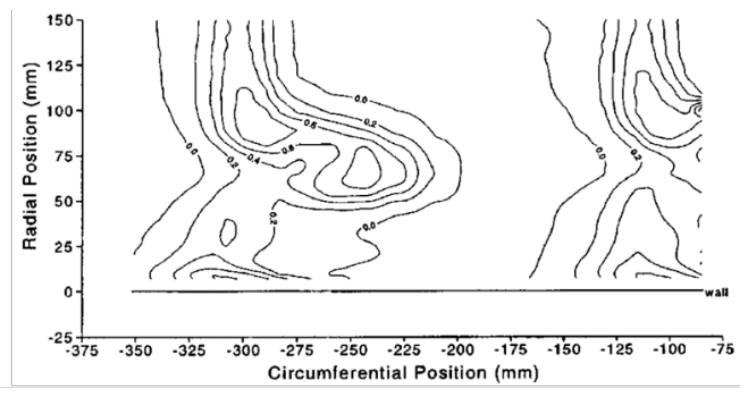

a) Experiment

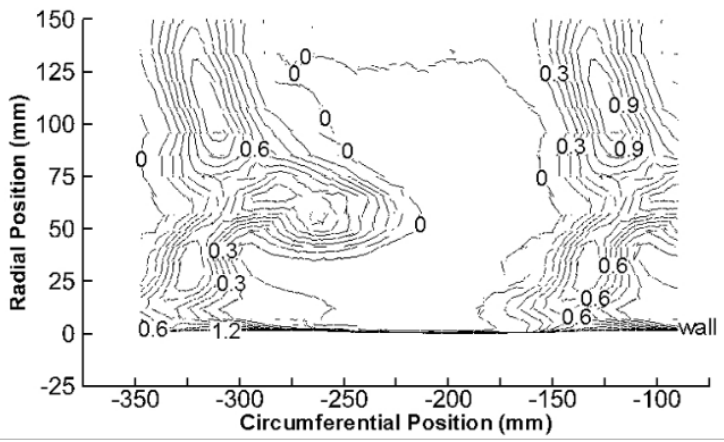

b) DG1 Laminar

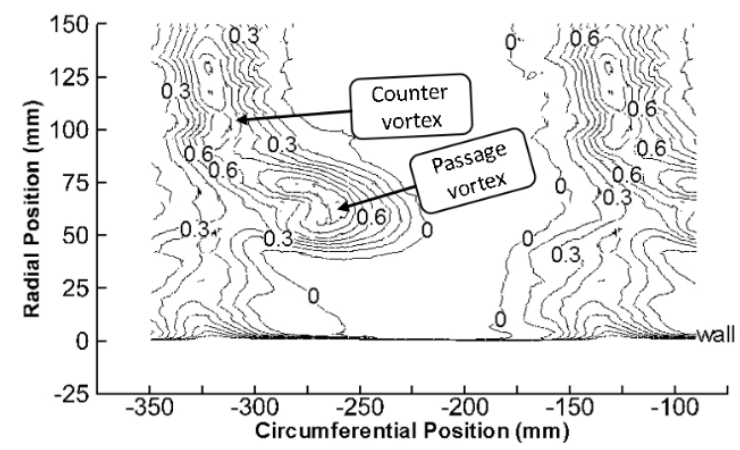

c) DG2 Laminar

Fig. 21. Total pressure loss coefficient $\mathrm{Cp} 0$ from direct laminar at plane $128 \% C_{a x}$

More quantitative comparisons can be made by means of the pitchwise averaged flow performance parameters. The pitchwise mass-averaged $\mathrm{Cp} 0$, yaw angle and secondary kinetic energy coefficient $\mathrm{C}_{\text {ske }}$ at position $128 \% C_{a x}$ are shown in Figs. 22 (a), 22(b) and 22(c) respectively. Compared to the RANS solution, the loss peak predicted by the present unsteady laminar solution shows a significant reduction, thus is 
much closer to the experimental value. Yet the peak moves towards the end wall by $5 \mathrm{~mm}$, this might well be attributed to lack of turbulence diffusion of the endwall flow as predicted by the pure laminar flow model. Near the midspan, the losses from the direct laminar are comparable to the experimental one. The yaw angle and secondary kinetic energy coefficient $C_{\text {ske }}$ from the direct laminar near the end wall are better predicted than the RANS in terms of the shapes of the spanwise variation of the experimental data.

(a)

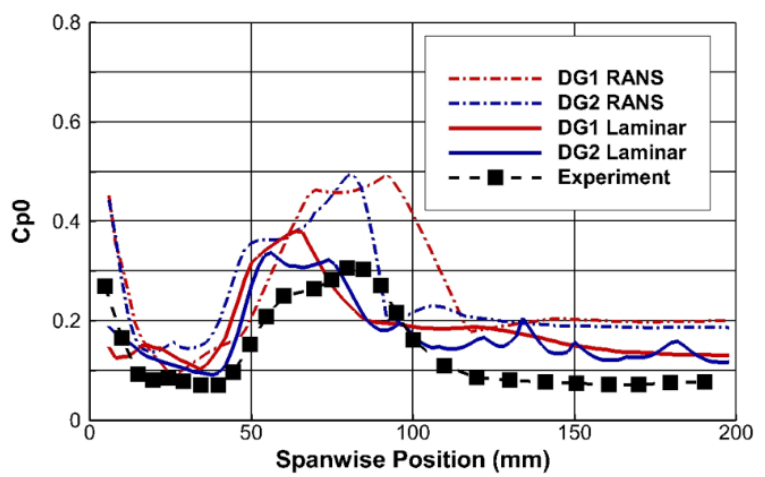

(b)

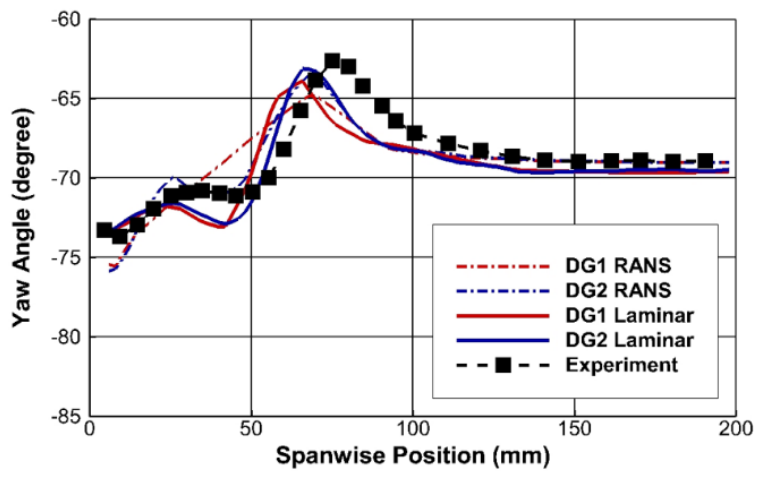

(c)

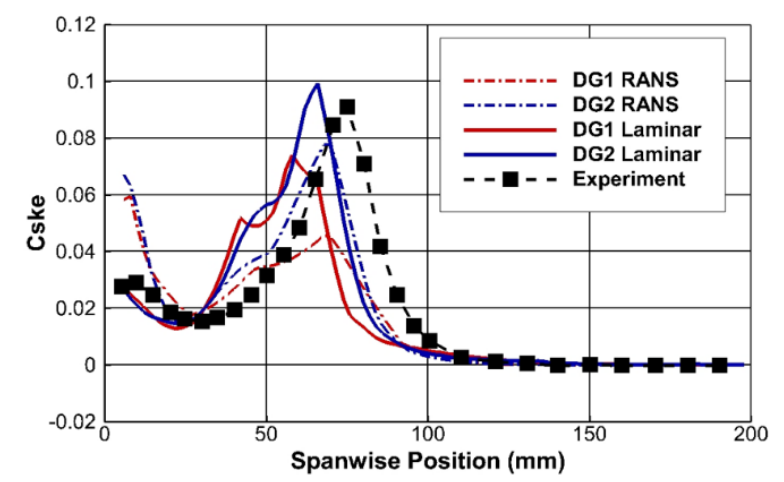

Fig. 22. Pitch-averaged results at plane $128 \% C_{a x}$ (a) total pressure loss coefficient Cp0

(b) yaw angle (c) secondary kinetic energy coefficient $C_{\text {ske }}$ 
The overall mass-averaged total pressure loss values at plane $128 \% C_{a x}$ are given in Table 4. The unsteady laminar solution shows a significant loss reduction compared to the RANS solution, and is much closer to the experimental value.

Table 4. Overall mass-averaged losses $\mathrm{Cp} 0$ at plane $128 \% C_{a x}$

\begin{tabular}{|c|c|c|c|c|}
\hline \multirow{2}{*}{ Experiment } & \multicolumn{2}{|c|}{ RANS } & \multicolumn{2}{c|}{ Laminar } \\
\cline { 2 - 5 } & DG1 & DG2 & DG1 & DG2 \\
\hline 0.136 & 0.257 & 0.245 & 0.178 & 0.166 \\
\hline
\end{tabular}

\section{CONCLUSIONS}

An implicit solver using the Discontinuous Galerkin discretization on unstructured meshes has been developed, validated and applied for analysis of turbine blade secondary flow. The DG scheme is distinctive in that a higher order accuracy is achieved by simple internal sub-divisions of a given mesh cell. This, in combination with the implicit time-integration formulation, is well suited for an unstructured mesh solver.

The preliminary test for a flow around cylinder is conducted to verify the method implementation, and also to illustrate the importance of a higher-order geometrical representation. A test for a flat plate turbulent boundary layer highlights the advantage of the simple internal sub-division of a mesh cell in relaxing the fine near wall mesh generation as required by a conventional low-order scheme.

The developed DG solver is then applied to a high pressure turbine cascade (Durham cascade) for which flow losses are strongly influenced by the boundary layer transition on the blade surface and the secondary flow development in the endwall 
region. The validation studies using the $2^{\text {nd }}$ and $3^{\text {rd }}$ order DG schemes based on the RANS with a one-equation turbulence model show consistent over-predictions of the flow losses, in line with previous RANS solutions. The loss overprediction is attributed to the transitional flow nature of the case, which is confirmed by further RANS simulations with a prescribed tripping point in line with the experimentally observed transition, in lieu of a validated transition model.

A more interesting and remarkable observation is that the flow losses predicted by a direct unsteady laminar solution are consistently better than those produced by the fully turbulent RANS solution. Furthermore, it is noted that straightforward unsteady laminar solutions with no empirical input seem to be comparable to (or slightly better than) the tripped RANS with empirical input. In relation to underlining flow physics as the different models predicted, a steady laminar solution seems to suppress instabilities of separated shear layer, leading to a very large separation bubble. The unsteady laminar solutions, on the other hand, show that unstable separated shear layers lead to coherently shed unsteady vortices with marked net entrainment from main stream to near-wall flow. The resultant time-averaged near-wall flow then seems to become effectively a reattached steady boundary layer. It should be pointed out that the present observation on the computed unsteady laminar separation behavior on a typical coarse RANS mesh for a turbine cascade is similar to that of a recent work for a compressor cascade using a different CFD solver [37]. Given the marked differences in the flow performance computed and the corresponding computational resources required, these findings should deserve further investigations. 


\section{NOMENCLATURE}

$\Omega \quad$ computational element

$\partial \Omega \quad$ boundary face

$\mathbf{x}$

spatial coordinates

$t \quad$ time

$\mathbf{U}$ state variables vector

$\mathbf{U}_{h} \quad$ approximate solution

$\mathbf{F}^{c} \quad$ inviscid flux

$\mathbf{F}^{v} \quad$ viscous flux

A Jacobian matrix of viscous flux

S source term

$h \quad$ finite element approximation (subscript)

$\mathbf{v}_{h} \quad$ test function

$\mathbf{V}_{h} \quad$ finite three-dimensional space

R residual vector

$b_{\text {ior } j} \quad$ basis shape function

M global mass matrix

$C_{a x} \quad$ axial chord

$\mathrm{Cp} \quad$ static pressure coefficient 


$$
\begin{array}{ll}
\text { Cp0 total pressure loss coefficient } \\
\text { Cske } & \text { Secondary kinetic energy coefficient }
\end{array}
$$

\section{ACKNOWLEDGMENT}

The first author acknowledges the support of the Computational Aerothermal Chair Studentship, Department of Engineering Science, University of Oxford and the support of China Scholarship Council (CSC).

\section{REFERENCES}

[1] Jimbo, T., Biswas, D., and Niizeki, Y., 2012. "Studies on unsteady flow characteristics in a high pressure turbine cascade based on a high-order large eddy simulation turbulence model". ASME J. Turbomach, 134(5), p. 051018.

[2] Marty, J., Lantos, N., Michel, B., and Bonneau, V., 2015. "LES and hybrid RANS/LES simulations of turbomachinery flows using high order methods". ASME Paper No. GT2015-42134.

[3] Read, W. H. and Hill, T. R., 1973. "Triangular Mesh Methods for the Neutron Transport Equations". Technical Report LA-UR-73-479, Los Alamos Scientific Laboratory.

[4] Cockburn, B. and Shu, C. W., 1998. "The Runge-Kutta Discontinuous Galerkin Method for Conservation Laws V: Multidimensional Systems". J. Comput. Phys., 141(2), pp. 199-224.

[5] Bassi, F., and Rebay, S., 1997. "A High-order Accurate Discontinuous Finite Element Method for the Numerical Solution of the Compressible Navier-Stokes Equations". J. Comput. Phys., 131(2), pp. 267-279. 
[6] Vincent, P. E., Castonguay, P. and Jameson, A., 2011. "A New Class of High-order Energy Stable Flux Reconstruction Schemes". J Sci Comput, 47, pp. 50-72.

[7] Bassi, F., Crivellini, A., Rebay, S., and Savini, M., 2005. "Discontinuous Galerkin solution of the reynolds-averaged Navier-Stokes and k-w turbulence model equations". Comput. Fluids, 34, pp. 507-540.

[8] Ghidoni, A., Colombo, A., Rebay, S., and Bassi, F., 2013. "Simulation of the transitional flow in a low pressure gas turbine cascade with a high-order discontinuous Galerkin method". ASME J. Fluids Eng., 135(7), p. 071101.

[9] Bassi, F., Botti, L., Colombo, A., A.Crivellini, Franchina, N., and Ghidoni, A., 2016. "Assessment of a high-order accurate discontinuous Galerkin method for turbomachinery flows". International Journal of Computational Fluid Dynamics, 30(4), pp. 307-328.

[10] Cui, J., Rao, V. N., and Tucker, P., 2017. "Numerical investigation of secondary flows in a high-lift low pressure turbine". International Journal of Heat and Fluid Flow, 63, pp. 149-157.

[11] Pichler, R., Zhao, Y., Sandberg, R., Michelassi, V., Pacciani, R., Marconcini, M., and Arnone, A., 2018. "LES and RANS Analysis of the End-Wall Flow in a Linear LPT Cascade: Part I - Flow and Secondary Vorticity Fields Under Varying Inlet Condition". ASME Paper No. GT2018-76233.

[12] Marconcini, M., Pacciani, R., Arnone, A., Michelassi, V., Pichler, R., Zhao, Y., and Sandberg, R., 2018. "LES and RANS Analysis of the End-Wall Flow in a Linear LPT Cascade With Variable Inlet Conditions: Part II - Loss Generation". ASME Paper No. GT201876450 .

[13] De Wiart, C. C., Hillewaert, K., and Geuzaine, P., 2012. “DNS of a low pressure turbine blade computed with the discontinuous Galerkin method". ASME Paper No. GT201268900.

[14] Hillewaert, K., de Wiart, C. C., Verheylewegen, G., and Arts, T., 2014. "Assessment of a high-order discontinuous Galerkin method for the direct numerical simulation of transition at low-Reynolds number in the T106C high-lift low pressure turbine cascade". ASME Paper No. GT2014-26739.

[15] De Wiart, C. C., Hillewaert, K., Lorriaux, E., and Verheylewegen, G., 2015. "Development of a discontinuous Galerkin solver for high-quality wallresolved/moddelled DNS and LES for practical turbomachinery flows on fully 
unstructured meshes". ASME Paper No. GT2015-43428.

[16] Garai, A., Diosady, L., Murman, S., and Madavan, N., 2015. "DNS of flow in a lowpressure turbine cascade using a discontinuous-Galerkin spectral-element method". ASME Paper No. GT2015-42773.

[17] Garai, A., Diosady, L. T., Murman, S. M., and Madavan, N. K., 2016. "DNS of lowpressure turbine cascade flows with elevated inflow turbulence using a discontinuousGalerkin spectral-element method". ASME Paper No. GT2016-56700.

[18] Denton, J. D., 1993. “Loss mechanisms in turbomachines". ASME J. Turbomach, 115(4), pp. 621-656.

[19] He, L., 1998. “Unsteady Flow in Oscillating Turbine Cascades: Part 2-Computational Study". ASME J. Turbomach, 120(2), pp. 269-275.

[20] Langtry, R. B., and Menter, F. R., 2009. "Correlation-Based Transition Modelling for Unstructured Parallelized Computational Fluid Dynamics Codes". AIAA J., 47(12), pp. 2894-2906.

[21] Liou, M. S., 2006. "A sequel to AUSM, Part II: AUSM+- up for all speeds". J. Comput. Phys., 214(1), pp. 137-170.

[22] Moro, D., Nguyen, N. C., and Peraire, J., 2011. "Navier-Stokes Solution Using Hybridizable Discontinuous Galerkin Methods". AIAA Paper No. 2011-3407.

[23] Persson, P. O., and Peraire, J., 2008. "Newton-GMRES preconditioning for discontinuous Galerkin discretizations of the Navier-Stokes equations". SIAM J. Sci. Comput., 30(6), pp. 2709-2733.

[24] Geuzaine, C., and Remacle, J. F., 2009. "Gmsh: a three-dimensional finite element mesh generator with built-in pre- and post-processing facilities". Int J Numer Meth Eng., 79(11), pp. 1309-1331.

[25] Landmann, B., 2008. "A parallel discontinuous Galerkin code for the Navier-Stokes and Reynolds-averaged Navier-Stokes equations". PhD thesis, University of Stuttgart.

[26] Thom, A., 1933. "The flow past circular cylinders at low speeds". Proc. R. Soc. Lond., Ser A. 141(845), pp. 651-669.

[27] He, L., 2017. "Multiscale Block Spectral solution for unsteady flows". Int J Numer Meth Fluids., 86(10), pp. 655-678. 
[28] Hartland, J. C., Gregory-Smith, D. G., Harvey, N. W., and Rose, M. G., 2000. "Nonaxisymmetric turbine end wall design: Part ii experimental validation". ASME J. Turbomach, 122(2), pp. 286-293.

[29] Gregory-Smith, D. G., Graves, C. P., and Walsh, J. A., 1988. "Growth of secondary losses and vorticity in an axial turbine cascade". ASME J. Turbomach, 110(1), pp. 1-8.

[30] Gregory-Smith, D. G. and Cleak, J. G. E., 1992. "Secondary Flow Measurements in a Turbine Cascade with High Inlet Turbulence". ASME J. Turbomach, 114(1), pp. 173-183.

[31] Cleak, J. G. E., and Gregory-Smith, D. G., 1992. "Turbulence modeling for secondary flow prediction in a turbine cascade". ASME J. Turbomach, 114(3), pp. 590-598.

[32] Ingram, G., Gregory-Smith, D., and Harvey, N., 2005. "Investigation of a Novel Secondary Flow Feature in a Turbine Cascade with End Wall Profiling". ASME J. Turbomach, 127(1), pp. 209-214.

[33] Moore, H., and Gregory-Smith, D. G., 1996. "Transition effects on secondary flows in a turbine cascade". ASME Paper No. 96-GT-100.

[34] Yao, M., and He, L., 2017. "High-order simulation of turbomachinery flow using a discontinuous Galerkin method". Global Power and Propulsion Society Conference, Paper No. 2017-0201.

[35] Kiran, K. N., and Anish, S., 2017. "An investigation on the effect of pitchwise endwall design in a turbine cascade at different incidence angles". Aerosp Sci Technol, 71, pp 382391.

[36] Ning, W., and He, L., 2001. "Some Modeling Issues on Trailing-Edge Vortex Shedding". AIAA J., 39(5), pp. 787-793.

[37] He, L., and Yi, J., 2017. "Two-scale methodology for URANS/Large Eddy Simulation solutions of unsteady turbomachinery flows". ASME J. Turbomach, 139(10), p. 101012.

[38] Hartland, J., 2001. "Secondary Loss Reduction in Rotor Blades by Non-Axisymmetric End-Wall Profiling". PhD thesis, University of Durham. 